\title{
ON LOG DEL PEZZO SURFACES IN LARGE CHARACTERISTIC
}

\author{
PAOLO CASCINI, HIROMU TANAKA, JAKUB WITASZEK
}

\begin{abstract}
We show that any Kawamata log terminal del Pezzo surface over an algebraically closed field of large characteristic is globally $F$-regular or it admits a log resolution which lifts to characteristic zero. As a consequence, we prove the KawamataViehweg vanishing theorem for klt del Pezzo surfaces of large characteristic.
\end{abstract}

\section{Contents}

1. Introduction

1.1. Sketch of the proof

2. Preliminaries

2.1. Log canonical surface singularities 6

2.2. Global F-adjunction 12

2.3. Perturbations 15

2.4. Flat families of log pairs and liftability 18

3. Proof of the main theorem for $\epsilon$-klt log del Pezzo

4. Liftability to characteristic zero 22

5. Non- $\epsilon$-klt case and proof of Theorem $1.1 \quad 24$

6. Kawamata-Viehweg vanishing for log del Pezzo surfaces 30

7. Example in characteristic two 34

References

\section{INTRODUCTION}

Recently, starting from the work of Hacon and Xu [HX15], many of the classical results of the minimal model programme in characteristic zero have been extended to three dimensional varieties over an algebraically closed field $k$ of characteristic $p>5$ [Bir16, CTX15, BW14].

2010 Mathematics Subject Classification. 14E30, 14F17, 13 A35.

Key words and phrases. Log del Pezzo surface, F-singularities, KawamataViehweg vanishing, positive characteristic.

All three authors were funded by EPSRC. 
One of the main tools used in [HX15] is the theory of $F$-singularities, replacing the use of the classical vanishing theorems, which hold only in characteristic zero (e.g. see Sch09]). Their proof of the existence of flips relies on the fact that if char $k>5$ and $(X, \Delta)$ is a one-dimensional projective klt pair such that $-\left(K_{X}+\Delta\right)$ is ample and the coefficients of $\Delta$ are contained in the standard set $\left\{1-\frac{1}{n} \mid n \in \mathbb{Z}_{>0}\right\}$, then $(X, \Delta)$ is globally F-regular [Wat91, Theorem 4.2]. Thus, it is natural to ask whether this result can be generalised to higher dimensional varieties. Unfortunately, in [CTW16] we give a negative answer to this question. Indeed, we show that over an arbitrary algebraically closed field $k$ of characteristic $p>0$, there exists a projective klt surface $X$ over $k$ such that $-K_{X}$ is ample, but $X$ is not globally $F$-regular. Thus, even in large characteristic, it is not known a priori whether klt del Pezzo surfaces admit desirable properties, such as the Kawamata-Viehweg vanishing theorem and the Bogomolov bound, which in particular gives a bound on the number of singular points on a klt del Pezzo surface of Picard number one ([Lan16] and [KM99, Section 9]).

The goal of this paper is to show the following:

Theorem 1.1. Let $I \subseteq(0,1) \cap \mathbb{Q}$ be a finite set.

Then there exists a positive integer $p(I)$ which satisfies the following property:

Let $(X, B)$ be a two dimensional projective klt pair over an algebraically closed field of characteristic $p>p(I)$ such that $-\left(K_{X}+B\right)$ is ample and the coefficients of $B$ are contained in $I$.

Then one of the following properties holds:

(1) $(X, B)$ is globally F-regular, or

(2) there exists a log resolution $\mu: V \rightarrow X$ of $(X, B)$ such that $\left(V, \operatorname{Exc}(\mu) \cup \mu_{*}^{-1}(\operatorname{Supp} B)\right)$ lifts to characteristic zero over a smooth base (cf. Definition 2.15).

Note that we do not know whether there exists a klt del Pezzo surface in large characteristic which violates (2) of Theorem 1.1.

Using the above result, the minimal model programme and the logarithmic version of a result of Deligne-Illusie [Har98, Corollary 3.8], we prove the Kawamata-Viehweg vanishing theorem for klt del Pezzo surfaces in large characteristic:

Theorem 1.2. There exists a positive integer $p_{0}$ which satisfies the following property:

Let $(X, \Delta)$ be a two dimensional projective klt pair over an algebraically closed field of characteristic $p>p_{0}$. Suppose that there exists 
an effective $\mathbb{Q}$-divisor $B$ such that $(X, B)$ is klt and $-\left(K_{X}+B\right)$ is nef and big. If $D$ is a $\mathbb{Z}$-divisor on $X$ such that $D-\left(K_{X}+\Delta\right)$ is nef and big, then

$$
H^{i}\left(X, \mathcal{O}_{X}(D)\right)=0 \quad \text { for any } i>0 .
$$

Note that Theorem 1.1 imposes a condition on the coefficients of the boundary divisor $B$, but Theorem 1.2 does not need such an assumption.

Finally, we show that Theorem 1.1 does not hold in characteristic two.

Theorem 1.3. There exists a projective klt surface $X$ over $\overline{\mathbb{F}}_{2}$ which satisfies the following properties:

(1) $-K_{X}$ is ample,

(2) $X$ is not globally $F$-split, and

(3) for any log resolution $h: W \rightarrow X$ of $X$, the pair $(W, \operatorname{Exc}(h))$ does not lift to characteristic zero over a smooth base (cf. Definition 2.15).

We prove Theorem 1.1 in Section 5, Theorem 1.2 in Section 6 and Theorem 1.3 in Section 7.

1.1. Sketch of the proof. We now give an overview of some of the steps of the proof of Theorem 1.1.

For simplicity, we assume that $I=\emptyset$. Let $X$ be a projective klt surface in large characteristic and such that $-K_{X}$ is ample. We want to show that at least one of the statements (1) and (2) in Theorem 1.1 holds true. The idea is that, choosing a suitable $\epsilon \in \mathbb{Q}_{>0}$, we consider two different cases:

(a) $X$ is $\epsilon$-klt.

(b) $X$ is not $\epsilon$-klt.

(a) It is known that, for a fixed algebraically closed field $k$, the set of all the $\epsilon$-klt del Pezzo surfaces over $k$ forms a bounded family [Ale94]. We generalise this boundedness result to mixed characteristic, i.e. there exists a projective morphism $\mathcal{X} \rightarrow S$ of schemes of finite type over Spec $\mathbb{Z}$, depending only on $\epsilon$, such that an arbitrary $\epsilon$-klt del Pezzo surface $X$ over an arbitrary algebraically closed field $k$ of characteristic $p>5$ is isomorphic to some fibre $\mathcal{X}_{s}$ up to a base change of the base field. By noetherian induction, we can show that for $p \gg 0$, any $\epsilon$ klt del Pezzo surface $X$ over an algebraic closed field of characteristic $p$ admits a $\log$ resolution $\mu: V \rightarrow X$ such that $(V, \operatorname{Exc}(\mu))$ lifts to characteristic zero over a smooth base. For more details, see Section 3 , 
(b) Assume that $X$ is not $\epsilon$-klt. In this case, the proof consists of the following three steps:

(I) Since $X$ is not $\epsilon$-klt, we can extract a prime divisor $C$ with $\log$ discrepancy $a:=a(C, X, 0) \leq \epsilon$ by:

$$
f: Y \rightarrow X, \text { so that } K_{Y}+(1-a) C=f^{*} K_{X} .
$$

(II) We run a $-\left(K_{Y}+C\right)$-MMP with scaling of $C$ :

$$
(Y, C)=:\left(Y_{0}, C_{0}\right) \rightarrow\left(Y_{1}, C_{1}\right) \rightarrow \cdots \rightarrow\left(Y_{n}, C_{n}\right)=:\left(Z, C_{Z}\right) .
$$

Note that since $(Y, \Delta)$ is $\log$ del Pezzo for some effective $\mathbb{Q}$ divisor $\Delta$, we may run a $D$-MMP for any $\mathbb{Q}$-Cartier divisor $D$. Indeed, let $s>0$ be a sufficiently small rational number such that $A:=s D-\left(K_{Y}+\Delta\right)$ is ample. Then,

$$
s D=K_{Y}+\Delta+A
$$

and in particular, a $D$-MMP coincides with a $\left(K_{Y}+\Delta+A\right)$ MMP. Furthermore, if $\epsilon$ is sufficiently small, then since $1-a \in$ $[1-\epsilon, 1)$, it follows that each pair $\left(Y_{i}, C_{i}\right)$ is $\log$ canonical by ACC for the log canonical threshold [Ale93, Theorem 1.1].

(III) We now consider five different cases as follows:

(i) $\kappa\left(Z,-\left(K_{Z}+C_{Z}\right)\right)=2$.

(ii) $\kappa\left(Z,-\left(K_{Z}+C_{Z}\right)\right)=1$.

(iii) $\kappa\left(Z,-\left(K_{Z}+C_{Z}\right)\right)=0$ and $\left(Z, C_{Z}\right)$ is not plt.

(iv) $\kappa\left(Z,-\left(K_{Z}+C_{Z}\right)\right)=0$ and $\left(Z, C_{Z}\right)$ is plt.

(v) $\kappa\left(Z,-\left(K_{Z}+C_{Z}\right)\right)=-\infty$.

If one of the cases (i), (ii), or (iii) holds, then we can show that $X$ is globally $F$-regular, i.e. (1) in Theorem 1.1 holds. If (iv) holds, then (2) in Theorem 1.1 holds. Finally, we show that (v) does not occur if $\epsilon$ is sufficiently small.

We now give some details of the methods we use in the steps above. After Step (I), there exists a rational number $b \in(1-a, 1)$, such that $(Y, b C)$ is klt and $-\left(K_{Y}+b C\right)$ is ample. The easiest case is if $-\left(K_{Y}+C\right)$ is ample and $\left(p^{e}-1\right)\left(K_{Y}+C\right)$ is Cartier for some positive integer $e$. In this case, Lemma 2.7 implies immediately that $\left(Z, C_{Z}\right)$ is globally $F$-split, which in turn implies that $X$ is globally $F$-regular. However, $-\left(K_{Y}+C\right)$ is not ample in general, but by running an MMP in Step (II), we can get closer to this situation. Indeed, in case (i) of Step (III), we have that $-\left(K_{Z}+C_{Z}\right)$ is nef and big, and after contracting the curves $\Gamma$ with $\left(K_{Z}+C_{Z}\right) \cdot \Gamma=0$, we may assume that $-\left(K_{Z}+C_{Z}\right)$ is ample. By using Lemma 2.7, we can show that $X$ is globally $F$-regular. We can apply a similar argument to show that $X$ is globally $F$-regular in cases (ii) and (iii) of Step (III). 
Let us assume now that (iv) of Step (III) holds. In this case, we first show that the set of pairs $\left(Z, C_{Z}\right)$ as in (iv) forms a bounded family. By noetherian induction, we show that for $p \gg 0$, any $\left(Z, C_{Z}\right)$ has a $\log$ resolution $\pi: W \rightarrow Z$ such that $\left(W, \pi_{*}^{-1} C_{Z} \cup \operatorname{Exc}(\pi)\right)$ lifts to characteristic zero over a smooth base. If $W$ already dominates $Y$, then the induced birational morphism $W \rightarrow X$ is a $\log$ resolution as in (2) of Theorem 1.1. In general, we cannot hope that $W$ dominates $Y$, however we can show that (2) of Theorem 1.1 holds after taking some blow-ups of $W$. For more details, we refer to Section 4, especially Lemma 4.3 .

Finally, let us assume that (v) of Step (III) holds. In particular, $Z$ admits a $-\left(K_{Z}+C_{Z}\right)$-negative Mori fibre space. Let us assume for simplicity that $\rho(Z)=1$ and $K_{Z}+C_{Z}$ is ample. Since $-\left(K_{Y}+b C\right)$ is ample, so is its push-forward $-\left(K_{Z}+b C_{Z}\right)$. Thus, we may find $b^{\prime} \in(b, 1)$ such that

$$
K_{Z}+b^{\prime} C_{Z} \equiv 0
$$

In particular, $b^{\prime} \in(1-\epsilon, 1)$, and after possibly replacing $\epsilon$ by a smaller number, we derive a contradiction thanks to ACC for the log CalabiYau pairs [Ale93, Theorem 5.3] (cf. Lemma 2.3).

Acknowledgement: We would like to thank P. Achinger, Y. Gongyo, Y. Kawamata, A. Langer, C. Liedtke, Z. Patakfalvi, A. Sannai, K. Schwede and S. Takagi for many useful discussions and comments. We would like to thank the referee for carefully reading our manuscript and for suggesting several improvements.

\section{Preliminaries}

If not stated otherwise, we work over an algebraically closed field $k$ of characteristic $p>0$. We say that $X$ is a variety over an algebraically closed field $k$, if $X$ is an integral scheme which is separated and of finite type over $k$. A curve is a variety of dimension one, and a surface is a variety of dimension two. We say that a scheme $X$ is normal if the local ring $\mathcal{O}_{X, x}$ is an integrally closed integral domain for any point $x \in X$. In particular, if $X$ is a noetherian normal scheme, then $\Gamma\left(U, \mathcal{O}_{X}\right)$ is an integrally closed integral domain for any irreducible affine open subset $U$ of $X$. Given a proper morphism $f: X \rightarrow Y$ between normal varieties, we say that two $\mathbb{Q}$-Cartier $\mathbb{Q}$-divisors $D_{1}, D_{2}$ on $X$ are numerically equivalent over $Y$, denoted $D_{1} \equiv_{f} D_{2}$, if their difference is numerically trivial on any fibre of $f$.

We refer to [KM98 for the classical definitions of singularities (e.g., $k l t, p l t, \log$ canonical) appearing in the minimal model programme. A $\log \operatorname{pair}(X, \Delta)$ consists of a normal variety $X$ and a $\mathbb{Q}$-divisor $\Delta \geq 0$ 
such that $K_{X}+\Delta$ is $\mathbb{Q}$-Cartier. Note that we always assume that a klt (resp. plt, $\log$ canonical) pair $(X, \Delta)$ is a $\log$ pair, and in particular $\Delta$ is an effective $\mathbb{Q}$-divisor. Given a $\log$ pair $(X, B)$ and a divisorial valuation $E$ over $X$, we denote by $a(E, X, B)$ the log discrepancy of $(X, B)$ with respect to $E$. For $\varepsilon>0$, we say that $(X, B)$ is $\varepsilon-k l t$ if $a(E, X, B)>\varepsilon$ for any divisorial valuation $E$ over $X$. A two dimensional projective log pair $(X, \Delta)$ is log del Pezzo if $(X, \Delta)$ is klt and $-\left(K_{X}+\Delta\right)$ is ample.

Given a subset $I \subseteq[0,1]$, we say that $I$ is an $A C C$ (resp. a $D C C$ ) set if it satisfies the ascending chain condition (resp. the descending chain condition). Given a subset $I \subseteq[0,1]$, we define:

$$
I_{+}:=\left\{\sum_{j=1}^{m} i_{j} \mid i_{j} \in I \text { for } j=1, \ldots, m\right\} \cap[0,1],
$$

and

$$
D(I):=\left\{\frac{m-1+f}{m} \mid m \in \mathbb{Z}_{>0}, f \in I_{+}\right\} \cap[0,1] .
$$

For the definitions of $F$-singularities, we refer to [SS10, Definition 3.1] and [CTW16, Definition 1.6].

2.1. Log canonical surface singularities. Let $X$ be a normal variety over an algebraically closed field $k$. Let $C$ be a prime divisor on $X$ such that $K_{X}+C$ is $\mathbb{Q}$-Cartier and let $C^{\prime} \rightarrow C$ be its normalisation. Then there exists an effective $\mathbb{Q}$-Cartier $\mathbb{Q}$-divisor Diff $C^{\prime}$ on $C^{\prime}$ such that

$$
\left.\left(K_{X}+C\right)\right|_{C^{\prime}}=K_{C^{\prime}}+\operatorname{Diff}_{C^{\prime}} .
$$

Moreover, if $(X, C)$ is $\log$ canonical, then the coefficients of Diff $C^{\prime}$ are standard coefficients, i.e. they are contained in the set $\{1\} \cup\left\{1-\frac{1}{m} \mid m \in\right.$ $\left.\mathbb{Z}_{>0}\right\}$ (see $\left[\mathrm{K}^{+} 92\right.$, Proposition-Definition 16.5] and [Kol13, Definition 4.2 and Proposition 4.5]).

Let $X$ be a normal surface. A singular point $q \in X$ is said to be cyclic if the exceptional locus of the minimal resolution of $X$ at $q$ is a chain of rational curves $C_{1}, \ldots, C_{m}$. We denote by $\Gamma_{q}$ the intersection matrix $\left(-C_{i} \cdot C_{j}\right)$ associated to $C_{1}, \ldots, C_{m}$. In particular, if $d$ is a positive integer, then there are only finitely many possibilities for $\Gamma_{q}$ so that $\operatorname{det} \Gamma_{q}=d$ [Kol13, pag. 116].

A singular point $q \in X$ is said to be dihedral if the exceptional locus of the minimal resolution of $X$ at $q$ is the union of rational curves $C_{1}, \ldots, C_{m}$, with $m \geq 4$, where $C_{1}, \ldots, C_{m-2}$ is a chain of curves and $C_{m-1}, C_{m}$ are (-2)-curves which meet $C_{m-2}$ transversally in two distinct points away from $C_{m-3} \cap C_{m-2}$ [Kol13, 3.35(3)]. 
Lemma 2.1. Let $f: X \rightarrow Y$ be a proper birational morphism between klt surfaces. If $L$ is a Cartier divisor on $X$ such that $L \equiv_{f} 0$, then there exists a Cartier divisor $L_{Y}$ on $Y$ such that $\mathcal{O}_{X}(L) \simeq \mathcal{O}_{X}\left(f^{*} L_{Y}\right)$. In particular, if $D$ is a Weil divisor on $Y$ such that $f^{*} D$ is Cartier, then $D$ is Cartier.

Proof. Since a klt surface is automatically quasi-projective (e.g. see Fuj12, Lemma 2.2]), by taking suitable compactifications, we may assume that $X$ and $Y$ are projective. Let $h: \tilde{X} \rightarrow X$ be the minimal resolution and let $g: \tilde{X} \rightarrow Y$ be the induced morphism. It is enough to show that there exists a Cartier divisor $L_{Y}$ on $Y$ such that $\mathcal{O}_{\tilde{X}}\left(h^{*} L\right) \simeq \mathcal{O}_{\tilde{X}}\left(g^{*} L_{Y}\right)$. Let $E$ be the sum of the exceptional prime divisors of $g$. By running a $\left(K_{\tilde{X}}+E\right)$-MMP over $Y$, we may assume that $f$ is a birational morphism between projective klt surfaces such that $\rho(X / Y)=1$.

By using the base point free theorem over surfaces [Tan15, Theorem 3.2 and Corollary 3.6], we may apply the same argument as [KM98, Theorem 3.7(4)] to conclude.

Lemma 2.2. Let $(X, C)$ be a two dimensional plt pair, where $C$ is a prime divisor. If $q \in C$ is a singular point of $X$, then $q$ is cyclic and the coefficient of $\operatorname{Diff}_{C}$ at $q$ is given by $1-\frac{1}{m_{q}}$, where $m_{q}:=\operatorname{det}\left(\Gamma_{q}\right)$. Further, for each Weil divisor $D$ on $X$, we have that $m_{q} D$ is Cartier around $q$.

Proof. The first part of the lemma follows from [Kol13, Theorem 3.36]. We are left to show that if $D$ is a Weil divisor on $X$, then $m_{q} D$ is Cartier at $q$. Let $f: Y \rightarrow X$ be the minimal resolution of $X$ at $q$. Then the exceptional divisor is a chain of rational curves $C_{1}, \ldots, C_{r}$. We may write

$$
f^{*} D=D_{Y}+\sum_{i=1}^{r} b_{i} C_{i},
$$

where $D_{Y}$ is the strict transform of $D$ in $Y$ and $b_{1}, \ldots, b_{r} \in \mathbb{Q}$. Thus, for any $j=1, \ldots, r$, we have

$$
\sum_{i=1}^{r} b_{i} C_{i} \cdot C_{j}=-D_{Y} \cdot C_{j} .
$$

Since $D_{Y} \cdot C_{j} \in \mathbb{Z}$, it follows that $m_{q} b_{i} \in \mathbb{Z}$ for each $i=1, \ldots, r$. Since $X$ is klt at $q$, Lemma 2.1 implies the claim.

We need the following version of ACC for the nef threshold in dimension one and two: 
Lemma 2.3. Let $I \subseteq(0,1] \cap \mathbb{Q}$ be a DCC set. Then there exists a finite subset $J \subseteq I$ which satisfies the following property:

Let $(X, B)$ be a projective log canonical pair and let $\pi: X \rightarrow T$ be a projective morphism onto a normal variety $T$ such that $\pi_{*} \mathcal{O}_{X}=\mathcal{O}_{T}$ and

- $\operatorname{dim} X \leq 2$,

- $\operatorname{dim} T<\operatorname{dim} X$

- the coefficients of $B$ belong to $I$, and

- $K_{X}+B \equiv_{\pi} 0$.

Then the coefficient of any $\pi$-horizontal component of $B$ belongs to $J$.

Proof. See [Ale93, Theorem 5.3] and [Bir16, Proposition 11.7].

To show Proposition 2.5, we need the following:

Lemma 2.4. Let $(X, \Delta)$ be a two dimensional klt pair such that $X$ is smooth away from a closed point $x \in X$. Let $f: Y \rightarrow X$ be the minimal resolution of $X$, with exceptional divisors $E_{1}, \ldots, E_{n}$ and let $a_{i}=a\left(E_{i}, X, \Delta\right)$. Assume that $n \geq 8$ and let $\sigma$ be a permutation of $\{1, \ldots, n\}$ such that

$$
a_{\sigma(1)} \leq a_{\sigma(2)} \leq \cdots \leq a_{\sigma(n)} .
$$

Let $\ell$ be a positive integer such that $\ell \Delta$ is a $\mathbb{Z}$-divisor and

$$
\ell a_{\sigma(i)} \in \mathbb{Z} \quad \text { for } i=1, \ldots, 8 .
$$

Then $2 \ell\left(K_{X}+\Delta\right)$ is Cartier.

Proof. We have

$$
K_{Y}+f_{*}^{-1} \Delta+\sum_{i=1}^{n}\left(1-a_{i}\right) E_{i}=f^{*}\left(K_{X}+\Delta\right) .
$$

Since $X$ is klt and $\ell f_{*}^{-1} \Delta$ is integral, by Lemma 2.1, it is enough to show that $2 \ell a_{j} \in \mathbb{Z}$ for any $j=1, \ldots, n$.

We may assume that $x \in X$ is a cyclic or a dihedral singularity, as otherwise, by the classification of two dimensional klt singularities, we have that $n \leq 8$ (e.g. see [KM98, Theorem 4.16]), and the claim follows immediately.

We first assume that $x \in X$ is a cyclic singularity and $E_{1}, \ldots, E_{n}$ is a chain of rational curves. For each $i \in\{2, \ldots, n-1\}$, by taking the intersection with $E_{i}$ on both sides of the equality above, we obtain

$$
\left(-E_{i}^{2}\right) a_{i}+f_{*}^{-1} \Delta \cdot E_{8}=a_{i-1}+a_{i+1} .
$$


In particular, the numbers $a_{1}, \ldots, a_{n}$ satisfy the following convexity inequality:

$$
a_{i} \leq \frac{a_{i-1}+a_{i+1}}{-E_{i}^{2}} \leq \frac{a_{i-1}+a_{i+1}}{2} .
$$

Thus, after possibly replacing $\sigma(1)$ by another index $j \in\{1, \ldots, n\}$ such that $a_{\sigma(1)}=a_{j}$, we may assume that $\sigma(2)$ is equal to $\sigma(1)-1$ or $\sigma(1)+1$, say $\sigma(1)+1$. In particular, $\ell a_{\sigma(1)}, \ell a_{\sigma(1)+1} \in \mathbb{Z}$.

Thus, for each $i=2, \ldots, n-1$, we have

$$
\left(-E_{i}^{2}\right) \ell a_{i}+\left(\ell f_{*}^{-1} \Delta\right) \cdot E_{i}=\ell a_{i-1}+\ell a_{i+1},
$$

and it follows inductively that $\ell a_{j} \in \mathbb{Z}$ for any $j=1, \ldots, n$. Thus, the claim follows.

Let us assume now that $x \in X$ is a dihedral singularity. Let $E_{n-1}$ and $E_{n}$ be two tails of self-intersection $(-2)$ and let $E_{1}, E_{2}, \ldots, E_{n-2}$ be the remaining chain of rational curves [Kol13, 3.35(3)], so that $E_{n-2}$ intersects $E_{n-1}$ and $E_{n}$. For $m \in\{n-1, n\}$, by taking the intersection with $E_{m}$ on both sides of the equality

$$
K_{Y}+f_{*}^{-1} \Delta+\sum_{i=1}^{n}\left(1-a_{i}\right) E_{i}=f^{*}\left(K_{X}+\Delta\right),
$$

we obtain

$$
2 a_{m}+f_{*}^{-1} \Delta \cdot E_{m}=a_{n-2}+1 .
$$

Thus, it is enough to show that $\ell a_{i} \in \mathbb{Z}$ for any $i=1, \ldots, n-2$. By assumption, it follows that

$$
\ell \min _{1 \leq i \leq n-2} a_{i}, \ell \min _{\substack{1 \leq i \leq n-2 \\ i \neq j_{1}}} a_{i} \in \mathbb{Z}
$$

for some $j_{1} \in\{1, \ldots, n-2\}$ such that $a_{j_{1}}=\min _{1 \leq i \leq n-2} a_{i}$. By applying the same argument as above to the chain $E_{1}, E_{2}, \ldots, E_{n-2}$, we obtain that $\ell a_{j} \in \mathbb{Z}$ for every $j \in\{1, \ldots, n-2\}$. Thus, the claim follows.

Proposition 2.5. Let $I \subseteq(0,1] \cap \mathbb{Q}$ be a DCC set. Then there exists a positive integer $m_{0}$ which satisfies the following property:

Let $(X, \Delta)$ be a two dimensional projective log canonical pair such that

- $X$ is klt,

- the coefficients of $\Delta$ are contained in I, and

- $K_{X}+\Delta \equiv 0$.

Then $m_{0}\left(K_{X}+\Delta\right)$ is Cartier. 
Proof. By Lemma 2.3, we may assume that $I$ is a finite set. Further, we may assume that $1 \in I$. Let $(X, \Delta)$ be a log pair as in the Proposition.

First, we show that there exists $m_{1} \in \mathbb{Z}_{>0}$, depending only on $I$, such that $m_{1}\left(K_{X}+\Delta\right)$ is Cartier around any closed point $q \in X$ such that $(X, \Delta)$ is not klt at $q$. By Lemma 2.1, after taking a dlt modification, we may assume that $(X, \Delta)$ is dlt at $q$. If the support of $\llcorner\Delta\lrcorner$ is singular at $q$, then $X$ is smooth at $q$ [KM98, Theorem 4.15(1)], and there is nothing to show. Thus, we may assume that $(X, \Delta)$ is plt around $q$. Let $C$ be the component of $\lfloor\Delta\rfloor$ containing $q$. Since $(X, \Delta)$ is plt around $q$, we know that $C$ is smooth, and so we may write

$$
K_{C}+\Delta_{C}=\left.\left(K_{X}+\Delta\right)\right|_{C} \equiv 0,
$$

where, by inversion of adjunction, $\Delta_{C}$ is a $\mathbb{Q}$-divisor on $C$ such that $\left(C, \Delta_{C}\right)$ is klt. By [MP04, Lemma 4.3], $\Delta_{C}$ has coefficients in $D(I)$. Since $D(I)$ is a DCC set [MP04, Lemma 4.4] and Diff ${ }_{C} \leq \Delta_{C}$, Lemma 2.3 implies that there are only finitely many possibilities for the coefficients of $\operatorname{Diff}_{C}$ at $q$, and the existence of $m_{1}$ follows from Lemma 2.2 .

Now we show that there exists $m_{2} \in \mathbb{Z}_{>0}$, depending only on $I$, such that $m_{2}\left(K_{X}+\Delta\right)$ is Cartier around any closed point $q \in X$ such that $(X, \Delta)$ is klt at $q$. Let $f: Y \rightarrow X$ be the minimal resolution at $q$. We have

$$
K_{Y}+f_{*}^{-1} \Delta+\sum_{i=1}^{n}\left(1-a_{i}\right) E_{i}=f^{*}\left(K_{X}+\Delta\right)
$$

where $E_{1}, \ldots, E_{n}$ are the $f$-exceptional prime divisors, ordered so that

$$
a_{1} \leq a_{2} \leq \cdots \leq a_{n}
$$

By Lemma 2.4, if $\ell$ is a positive integer such that

$$
\ell a_{1}, \ell a_{2}, \ldots, \ell a_{8} \in \mathbb{Z}
$$

and such that $\ell \Delta$ is a $\mathbb{Z}$-divisor, then $2 \ell\left(K_{X}+\Delta\right)$ is Cartier around $q$. Thus, it is enough to find $n_{0} \in \mathbb{Z}_{>0}$, depending only on $I$, such that $n_{0} a_{1}, \ldots, n_{0} a_{8} \in \mathbb{Z}$.

To this end, we first extract a prime divisor $E_{1}$ such that $a\left(E_{1}, X, \Delta\right)=$ $a_{1}$ and we obtain a birational morphism $g: Z \rightarrow X$ such that

$$
K_{Z}+g_{*}^{-1} \Delta+\left(1-a_{1}\right) E_{1}=g^{*}\left(K_{X}+\Delta\right) \equiv 0 .
$$

By ACC for the minimal log discrepancy [Ale93, Theorem 3.2], the set $\left\{a_{1}\right\}_{(X, \Delta), x \in X}$ is an ACC set, hence $\left\{1-a_{1}\right\}$ is a DCC set. By Lemma 2.3, there are finitely many possibilities for $a_{1}$. Then, we extract $E_{2}$ with $a\left(E_{2}, X, \Delta\right)=a_{2}$, and apply the same argument, to show that there are only finitely many possibilities for $a_{2}$. Repeating the same 
argument eight times, we see that there are finitely many possibilities for $a_{1}, \ldots, a_{8}$. Thus, we may find a positive integer $n_{0}$ as above and the claim follows.

The following result is well known at least in characteristic zero [Pro01, Lemma 8.3.15]. We include the proof for the sake of completeness.

Lemma 2.6. Let $(Z, C+B)$ be a two dimensional $\mathbb{Q}$-factorial log canonical pair, where $C$ is a prime divisor and $B$ is an effective $\mathbb{Q}$ divisor. Let $\pi: Z \rightarrow T$ be a projective morphism onto a smooth curve T such that $\pi_{*} \mathcal{O}_{Z}=\mathcal{O}_{T}$. Assume that

- $C$ is $\pi$-horizontal,

- $K_{Z}+C+B \equiv_{\pi} 0$, and

- $\rho(Z / T)=1$.

Then the following hold:

(1) For every closed point $t \in T, \pi^{-1}(t)_{\text {red }}$ is isomorphic to $\mathbb{P}^{1}$.

(2) $[K(C): K(T)] \leq 2$.

(3) Assume that $C$ is normal, and let us define $K_{C}+B_{C}=\left(K_{Z}+\right.$ $C+B)\left.\right|_{C}$ by adjunction. If $[K(C): K(T)]=2$ and the field extension $K(C) / K(T)$ is separable, then $B_{C}$ is invariant under the action of the Galois group $\operatorname{Gal}(K(C) / K(T))$.

Proof. Let $F:=\pi^{-1}(t)_{\text {red. }}$. Note that $F$ is irreducible, because $Z$ is $\mathbb{Q}$-factorial and $\rho(Z / T)=1$. Since $C$ is $\pi$-horizontal, we obtain

$$
\left(K_{Z}+F\right) \cdot F=K_{Z} \cdot F<\left(K_{Z}+C+B\right) \cdot F=0 .
$$

Thus, [Tan14, Theorem 3.19(1)] implies (1).

Let $G$ be a general fibre of $\pi$. Then $G$ is integral [Băd01, Corollary 7.3]. Thus, $G \simeq \mathbb{P}^{1}$ by (1). In particular, it follows that

$$
\left(K_{Z}+B\right) \cdot G \geq K_{Z} \cdot G=\left(K_{Z}+G\right) \cdot G=\operatorname{deg}\left(K_{G}+\operatorname{Diff}_{G}\right) \geq-2,
$$

which implies $C \cdot G=-\left(K_{Z}+B\right) \cdot G \leq 2$. Thus, (2) holds.

We now prove (3). Note that $B$ is $\pi$-vertical, since

$$
0=\left(K_{Z}+C+B\right) \cdot G \geq-2+2+B \cdot G=B \cdot G,
$$

where $G$ is a general fibre of $\pi$. Since $\rho(Z / T)=1$, we obtain $K_{Z}+C \equiv_{\pi}$ 0 . Since the divisor $\left.\pi^{*}(t)\right|_{C}$ is $\operatorname{Gal}(K(C) / K(T))$-invariant for every closed point $t \in T$, so is $\left.B\right|_{C}$. In particular, we may assume $B=0$ and $B_{C}=\operatorname{Diff}_{C}$.

Let $Q \in T$ be such that $\pi^{-1}(Q) \cap C$ consists of two distinct points $Q_{1}, Q_{2}$. It is enough to show that the coefficients of Diff $C$ at the points $Q_{1}$ and $Q_{2}$ coincide. We may write $\pi^{*} Q=m F$ where $F$ is a prime divisor and $m$ is a positive integer. By $(1), F \simeq \mathbb{P}^{1}$. 
We show that the pair $(Z, C+F)$ is $\log$ canonical. We have

$$
\left.0 \sim_{\mathbb{Q}}\left(K_{Z}+C+F\right)\right|_{F}=K_{F}+\operatorname{Diff}_{F}+\left.C\right|_{F} .
$$

Since $(Z, C+F)$ is not plt at the points $Q_{1}$ and $Q_{2}$, inversion of adjunction implies that the pair $\left(F, \operatorname{Diff}_{F}+\left.C\right|_{F}\right)$ is not klt at the points $Q_{1}$ and $Q_{2}$. Thus,

$$
\operatorname{Diff}_{F}+\left.C\right|_{F} \geq Q_{1}+Q_{2}
$$

and since $\operatorname{deg}\left(\operatorname{Diff}_{F}+\left.C\right|_{F}\right)=\operatorname{deg}\left(-K_{F}\right)=2$, equality holds. Thus, inversion of adjunction implies that $(Z, C+F)$ is $\log$ canonical.

Since $\left.\pi\right|_{C}: C \rightarrow T$ is étale over $Q$, we have $\left.m F\right|_{C}=\left.\pi^{*} Q\right|_{C}=Q_{1}+Q_{2}$ and

$$
\left.\left(K_{Z}+C+F\right)\right|_{C}=K_{C}+\operatorname{Diff}_{C}+\frac{1}{m}\left(Q_{1}+Q_{2}\right) .
$$

In particular, by inversion of adjunction again, the coefficients of Diff ${ }_{C}$ at $Q_{1}$ and $Q_{2}$ are equal to $1-\frac{1}{m}$, and the claim follows.

2.2. Global F-adjunction. We now summarise some known results on $F$-adjunction which we will use in this subsection. Let $X$ be a normal variety, $S$ a prime divisor and $B$ an effective $\mathbb{Q}$-divisor on $X$ such that $S \nsubseteq \operatorname{Supp} B$. Let $\nu: S^{\prime} \rightarrow S$ be the normalisation. Assume that $\left(p^{e}-1\right)\left(K_{X}+S+B\right)$ is Cartier for some $e \in \mathbb{Z}_{>0}$ (this is equivalent to saying that the Cartier index of $K_{X}+S+B$ is not divisible by $p$ ). By adjunction, we may write

$$
\nu^{*}\left(\left.\left(K_{X}+S+B\right)\right|_{S}\right)=K_{S^{\prime}}+B_{S^{\prime}}
$$

(cf. Subsection 2.1). There are natural homomorphisms Sch14, discussion below Proposition 2.5]

$$
\phi_{S+B}^{X}: F_{*}^{e} \mathcal{O}_{X}\left(-\left(p^{e}-1\right)\left(K_{X}+S+B\right)\right) \rightarrow \mathcal{O}_{X},
$$

and

$$
\phi_{B_{S^{\prime}}}^{S^{\prime}}: F_{*}^{e} \mathcal{O}_{S^{\prime}}\left(-\left(p^{e}-1\right)\left(K_{S^{\prime}}+B_{S^{\prime}}\right)\right) \rightarrow \mathcal{O}_{S^{\prime}}
$$

Let

$$
\mathcal{L}:=\mathcal{O}_{X}\left(-\left(p^{e}-1\right)\left(K_{X}+S+B\right)\right) .
$$

Since $S$ is an $F$-pure centre of $(X, S+B)$ [ibid, Definition 5.1], we obtain a commutative diagram [ibid, Remark 5.2]:

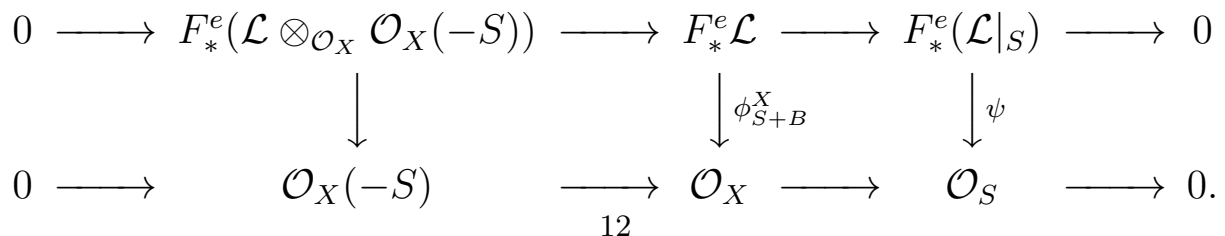


We also obtain the following commutative diagram [Sch09, Lemma 8.1]:

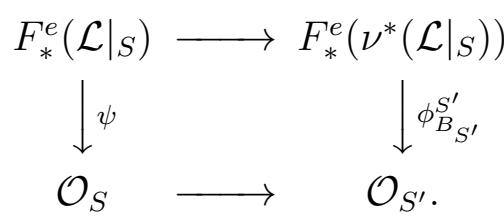

Note that the right vertical arrow coincides with $\phi_{B_{S^{\prime}}}^{S^{\prime}}$ by [Das15, Theorem 5.3].

Under some additional assumptions, we will show that if $-\left(K_{X}+S+\right.$ $B)$ is nef and $\left(S^{\prime}, B_{S^{\prime}}\right)$ is globally $F$-split, then $(X, S+B)$ is globally $F$-split as well. The following three lemmas correspond to the cases

$$
\kappa\left(X,-\left(K_{X}+S+B\right)\right)=2,1 \text { and } 0
$$

respectively.

Lemma 2.7. Let $(X, S+B)$ be a projective log pair where $S$ is a prime divisor and $B$ is an effective $\mathbb{Q}$-divisor such that $S \nsubseteq \operatorname{Supp} B$. Assume that

(1) $-\left(K_{X}+S+B\right)$ is ample,

(2) $S$ is normal and if $K_{S}+B_{S}=\left.\left(K_{X}+S+B\right)\right|_{S}$ is defined by adjunction, then $\left(S, B_{S}\right)$ is globally $F$-split, and

(3) $\left(p^{e}-1\right)\left(K_{X}+S+B\right)$ is Cartier for some positive integer $e$.

Then $(X, S+B)$ is globally $F$-split.

Proof. Let $\mathcal{L}=\mathcal{O}_{X}\left(-\left(p^{e}-1\right)\left(K_{X}+S+B\right)\right)$. We have the following diagram:

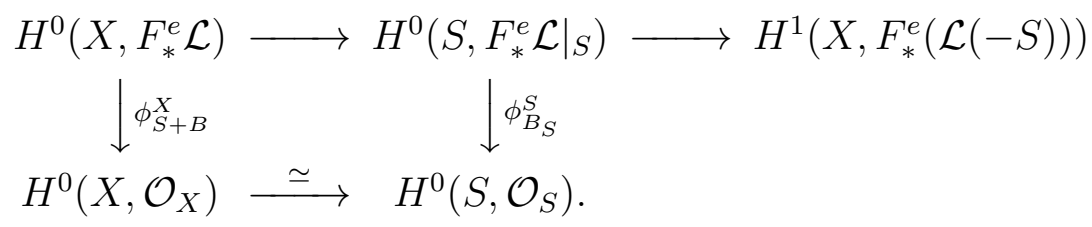

(2) implies that the right vertical arrow $\phi_{B_{S}}^{S}$ is surjective. By Serre vanishing, after possibly replacing $e$ by a larger multiple, (1) implies that $H^{1}\left(X, F_{*}^{e}(\mathcal{L}(-S))\right)=0$. By a diagram chase, it follows that $\phi_{S+B}^{X}$ is surjective. Thus, $(X, S+B)$ is globally $F$-split.

Lemma 2.8. Assume that char $k>2$. Let $(Z, C+B)$ be a two dimensional projective $\mathbb{Q}$-factorial log canonical pair, where $C$ is a prime divisor and $B$ is an effective $\mathbb{Q}$-divisor. Let $\pi: Z \rightarrow T$ be a morphism onto a smooth projective curve $T$ such that $\pi_{*} \mathcal{O}_{Z}=\mathcal{O}_{T}$. Assume that

(1) $C$ is $\pi$-horizontal,

(2) $-\left(K_{Z}+C+B\right) \equiv \pi^{*} A$ for some ample $\mathbb{Q}$-divisor $A$ on $T$, 
(3) $C$ is normal and if $K_{C}+B_{C}=\left.\left(K_{Z}+C+B\right)\right|_{C}$ is defined by adjunction, then $\left(C, B_{C}\right)$ is globally $F$-split,

(4) $\left(p^{e}-1\right)\left(K_{Z}+C+B\right)$ is Cartier for some positive integer e, and

(5) $\rho(Z / T)=1$.

Then $(Z, C+B)$ is globally $F$-split.

Proof. Let $\mathcal{L}:=\mathcal{O}_{Z}\left(-\left(p^{e}-1\right)\left(K_{Z}+C+B\right)\right)$. We have the following commutative diagram:

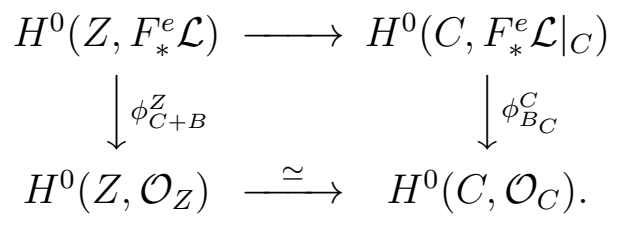

By (3), the right vertical arrow $\phi_{B_{C}}^{C}$ is surjective.

By (2) and (4), we can find an ample Cartier divisor $A^{\prime}$ on $T$ such that $\mathcal{L} \simeq \mathcal{O}_{Z}\left(\pi^{*} A^{\prime}\right)$ [Tan15, Theorem 0.4]. Since $\pi_{*} \mathcal{O}_{Z}=\mathcal{O}_{T}$, we have that

$$
H^{0}(Z, \mathcal{L}) \simeq H^{0}\left(T, \mathcal{O}_{T}\left(A^{\prime}\right)\right)
$$

and so the image of the upper horizontal arrow is exactly the $G$ invariant part: $H^{0}\left(C,\left.F_{*}^{e} \mathcal{L}\right|_{C}\right)^{G}$, where $G$ is the Galois group of $K(C) / K(T)$.

Let $f \in H^{0}\left(C,\left.F_{*}^{e} \mathcal{L}\right|_{C}\right)$ be such that $\phi_{B_{C}}^{C}(f)=1$. Since $B_{C}$ is $G$ invariant by Lemma 2.6, we have that $\phi_{B_{C}}^{C}$ is $G$-equivariant. In particular,

$$
\phi_{B_{C}}^{C}\left(\frac{1}{|G|} \sum_{g \in G} g(f)\right)=1 .
$$

We can divide by $|G|$, because char $k>2$ and $|G| \leq 2$ by Lemma 2.6. Thus, $\phi_{C+B}^{Z}$ is surjective and $(Z, C+B)$ is globally $F$-split.

Lemma 2.9. Let $(Z, C+B)$ be an $n$-dimensional projective log canonical pair with $n \leq 2$, where $C$ is a prime divisor and $B$ is an effective $\mathbb{Q}$-divisor. Assume that

(1) $\left(p^{e}-1\right)\left(K_{Z}+C+B\right) \sim 0$ for some positive integer $e$,

(2) $H^{1}\left(Z, \mathcal{O}_{Z}\right)=0$, and

(3) if $n=2$, then $(Z, C+B)$ is not plt.

Then $(Z, C+B)$ is globally F-split.

Proof. We only prove the case $n=2$, as the case $n=1$ is easier.

Let $\nu: C^{\prime} \rightarrow C$ be the normalisation. We may write

$$
\nu^{*}\left(\left.\left(K_{Z}+C+B\right)\right|_{C}\right)=K_{C^{\prime}}+B_{C^{\prime}}
$$

for some effective $\mathbb{Q}$-divisor $B_{C^{\prime}}$ on $C^{\prime}$ (cf. Subsection 2.1). By (3) and by inversion of adjunction, it follows that $\left\llcorner B_{C^{\prime}}\right\lrcorner \neq 0$. Thus, by (1), it 
follows that $C^{\prime}$ is isomorphic to $\mathbb{P}^{1}$. Therefore, by the same Lemma in dimension one, $\left(C^{\prime}, B_{C^{\prime}}\right)$ is globally $F$-split.

Let $e$ be a positive integer satisfying (1) and let

$$
\mathcal{L}:=\mathcal{O}_{Z}\left(-\left(p^{e}-1\right)\left(K_{Z}+C+B\right)\right) .
$$

By (1), we have that $\mathcal{L} \simeq \mathcal{O}_{Z}$. We consider the commutative diagram

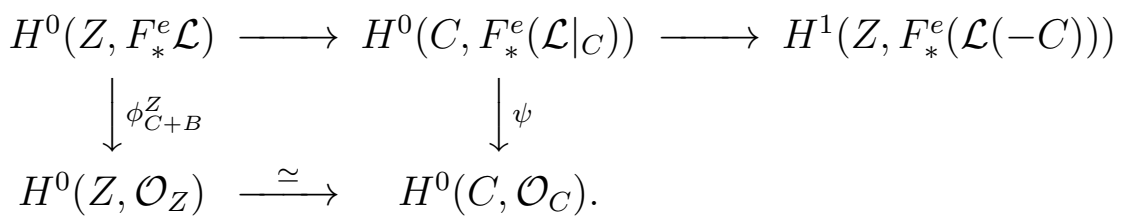

We now show that $\psi$ is surjective. We have the following commutative diagram:

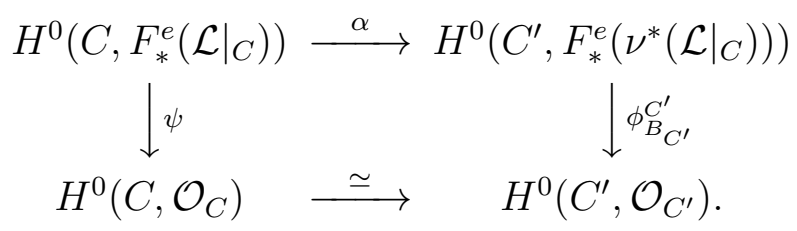

Since $\alpha$ is induced from the natural homomorphism $\mathcal{O}_{C} \rightarrow \nu_{*} \mathcal{O}_{C^{\prime}}$ and $\mathcal{L} \simeq \mathcal{O}_{Z}$, it follows that $\alpha$ is an isomorphism. Since $\left(C^{\prime}, B_{C^{\prime}}\right)$ is globally $F$-split, $\phi_{B_{C^{\prime}}}^{C^{\prime}}$ is surjective. Thus, $\psi$ is surjective as well.

Since $\mathcal{L} \simeq \mathcal{O}_{Z},(2)$ implies

$$
\begin{aligned}
H^{1}\left(Z, F_{*}^{e}(\mathcal{L}(-C))\right) & \simeq H^{1}(Z, \mathcal{L}(-C)) \\
& \simeq H^{1}\left(Z, \mathcal{O}_{Z}(-C)\right) \hookrightarrow H^{1}\left(Z, \mathcal{O}_{Z}\right)=0 .
\end{aligned}
$$

Since $\psi$ is surjective, by a diagram chase it follows that $\phi_{C+B}^{Z}$ is surjective as well, and, in particular, $(Z, C+B)$ is globally $F$-split. Thus, the claim follows.

2.3. Perturbations. Let $(X, S+B)$ be a log pair such that $S$ is a prime divisor and $B$ is an effective $\mathbb{Q}$-divisor such that $S \nsubseteq \operatorname{Supp} B$. If the Cartier index of $K_{X}+S+B$ is divisible by $p$, then, in order to apply the results from the previous subsection, we need to perturb the coefficients of $B$.

We begin with the following well known result:

Lemma 2.10. Let $(X, \Delta)$ be a quasi-projective globally sharply $F$-split pair. Then there exists an effective $\mathbb{Q}$-divisor $H$ such that $(X, \Delta+H)$ is globally sharply F-split and

$$
\left(p^{e}-1\right)\left(K_{X}+\Delta+H\right) \sim 0
$$

for some positive integer e. 
Proof. This follows from the same proof as [SS10, Theorem 4.3(ii)].

Lemma 2.11. Let $(X, \Delta)$ be a two-dimensional quasi-projective sharply $F$-pure log pair. Then there exists an effective $\mathbb{Q}$-divisor $H$ such that $(X, \Delta+H)$ is sharply F-pure, and

$$
\left(p^{e}-1\right)\left(K_{X}+\Delta+H\right)
$$

is Cartier for some positive integer $e$.

Proof. Let $U \subseteq X$ be an affine open subset such that none of the irreducible components of $\Delta$ are contained in $X \backslash U$ and such that, around each point of $X \backslash U$, we have that $X$ is smooth, the support of $\Delta$ is simple normal crossing and $(X, \Delta)$ is plt. Since $(X, \Delta)$ is sharply $F$-pure and $U$ is affine, it follows that $\left(U,\left.\Delta\right|_{U}\right)$ is globally sharply $F$ split.

By Lemma 2.10, there exists an effective $\mathbb{Q}$-divisor $H^{\prime}$ on $X$ such that $\left(U,\left.\left(\Delta+H^{\prime}\right)\right|_{U}\right)$ is globally sharply $F$-split and

$$
\left(p^{e}-1\right)\left(K_{U}+\left.\left(\Delta+H^{\prime}\right)\right|_{U}\right) \sim 0,
$$

for some positive integer $e$. Since $X$ is smooth around each point of $X \backslash U$, it follows that $\left(p^{e}-1\right)\left(K_{X}+\Delta+H^{\prime}\right)$ is Cartier on $X$. For every positive integer $d$, we have

$$
\left(K_{X}+\Delta+H^{\prime}\right)-\left(K_{X}+\Delta+\frac{1}{p^{d}+1} H^{\prime}\right)=\frac{p^{d}}{p^{d}+1} H^{\prime} .
$$

Thus, for every sufficiently large positive integer $d$, there exists $e(d) \in$ $\mathbb{Z}_{>0}$ such that

is Cartier.

$$
\left(p^{e(d)}-1\right)\left(K_{X}+\Delta+\frac{1}{p^{d}+1} H^{\prime}\right)
$$

Let $H:=\frac{1}{p^{d}+1} H^{\prime}$. It is enough to show that, after possibly replacing $d$ by a larger value, the pair $(X, \Delta+H)$ is sharply $F$-pure around any point $q \in X \backslash U$. By assumption, around $q$ we have that $X$ is smooth, the support of $\Delta$ is simple normal crossing and $(X, \Delta)$ is plt. Thus, if $q$ is not contained in the support of $\llcorner\Delta\lrcorner$ and $d$ is sufficiently large, then $(X, \Delta+H)$ is strongly $F$-regular. On the other hand, if $q$ is contained in the support of $\llcorner\Delta\lrcorner$ then the claim follows from inversion of adjunction (e.g. see [Sch09, Main Theorem], [Das15, Theorem A]).

Lemma 2.12. Assume that $\operatorname{char} k>2$. Let $(Z, C+B)$ be a two dimensional projective sharply $F$-pure pair, where $C$ is a prime divisor and $B$ is an effective $\mathbb{Q}$-divisor. Let $\pi: Z \rightarrow T$ be a morphism onto a smooth projective curve $T$ such that $\pi_{*} \mathcal{O}_{Z}=\mathcal{O}_{T}$. Assume that

(1) $(Z, B)$ is klt, 
(2) $-\left(K_{Z}+C+B\right) \equiv \pi^{*} A$ for some ample $\mathbb{Q}$-divisor $A$ on $T$,

(3) $C$ is $\pi$-horizontal,

(4) $\rho(Z / T)=1$, and

(5) $\left(p^{d}-1\right) B$ is a $\mathbb{Z}$-divisor for some positive integer $d$.

Then there exists an effective $\mathbb{Q}$-divisor $E$, whose support is contained in some fibres of $\pi$, such that $(Z, C+B+E)$ is $\log$ canonical and

$$
\left(p^{e}-1\right)\left(K_{Z}+C+B+E\right)
$$

is Cartier for some positive integer e.

Proof. By Lemma [2.6, there exists a non-empty open subset $T^{0} \subseteq T$ such that the induced morphism $\pi^{-1}\left(T^{0}\right) \rightarrow T^{0}$ is a $\mathbb{P}^{1}$-bundle. By (2) and (5), it follows that, after possibly shrinking $T^{0}$, there exists a positive integer $d$ such that

$$
\left.\left(p^{d}-1\right)\left(K_{Z}+C+B\right)\right|_{\pi^{-1}\left(T^{0}\right)} \sim 0 .
$$

By (1) of Lemma 2.6, every fibre of $\pi$ is irreducible. Thus, we may write

$$
\left(p^{d}-1\right)\left(K_{Z}+C+B\right) \sim \sum_{i=1}^{r} a_{i} F_{i}+\sum_{j=1}^{s} b_{j} F_{j}^{\prime},
$$

where $a_{i}, b_{j} \in \mathbb{Z}, F_{i}=\pi^{-1}\left(t_{i}\right)_{\mathrm{red}}$, and $F_{j}^{\prime}=\pi^{-1}\left(t_{j}^{\prime}\right)_{\mathrm{red}}$, with

$$
t_{1}, \ldots, t_{r}, t_{1}^{\prime}, \ldots, t_{s}^{\prime} \in T
$$

so that $(Z, C+B)$ is plt (resp. not plt) along $F_{i}$ (resp. at some point of $\left.F_{j}^{\prime}\right)$. Since $\llcorner C+B\lrcorner=C$ is $\pi$-horizontal and $(Z, B)$ is klt, by inversion of adjunction we can find $\alpha_{i} \in \mathbb{Q}_{>0}$ for $i=1, \ldots, r$ such that $(Z, C+$ $\left.B+\sum_{i=1}^{r} \alpha_{i} F_{i}\right)$ is $\log$ canonical and $\left(p^{e_{1}}-1\right)\left(K_{Z}+C+B+\sum_{i=1}^{r} \alpha_{i} F_{i}\right)$ is Cartier around $\bigcup_{i=1}^{r} F_{i}$ for some $e_{1} \in \mathbb{Z}_{>0}$.

Fix an index $1 \leq j \leq s$. It is enough to find $e_{2} \in \mathbb{Z}_{>0}$ such that $\left(p^{e_{2}}-1\right) b_{j} F_{j}^{\prime}$ is Cartier. By construction, there exists a zero-dimensional $\log$ canonical centre $z \in F_{j}^{\prime}$ of $(Z, C+B)$. Since $(Z, B)$ is klt, it follows that $z \in C$. Let $m_{z}$ be the Cartier index of $F_{j}^{\prime}$ at $z$. Since $z \in C$, it follows that $m_{z} F_{j}^{\prime} \cdot C \geq 1$. We can write $\pi^{*}(\pi(z))=m F_{j}^{\prime}$ for some positive integer $m$. Lemma 2.6 implies that $m F_{j}^{\prime} \cdot C=\pi^{*}(\pi(z)) \cdot C \leq 2$. Thus,

$$
\frac{1}{m_{z}} \leq F_{j}^{\prime} \cdot C \leq \frac{2}{m}
$$

and in particular $2 m_{z} \geq m$. Since $m_{z}$ divides $m$, it follows that either $m=m_{z}$ or $m=2 m_{z}$.

By applying Lemma 2.10 to an affine open neighbourhood $U$ of $z$, we can find $e_{3} \in \mathbb{Z}_{>0}$ and an effective $\mathbb{Q}$-divisor $H$ on $U$ such that 
$\left(U,\left.(C+B)\right|_{U}+H\right)$ is sharply $F$-pure and

$$
\left(p^{e_{3}}-1\right)\left(K_{U}+\left.(C+B)\right|_{U}+H\right) \sim 0 .
$$

Since $z \in Z$ is a zero-dimensional $\log$ canonical centre of $(Z, C+B)$, it follows that $z$ is not contained in the support of $H$. Therefore, $\left(p^{e_{3}}-1\right)\left(K_{Z}+C+B\right)$ is Cartier around $z$. Since

$$
\left(p^{d}-1\right)\left(K_{Z}+C+B\right) \sim \sum_{i=1}^{r} a_{i} F_{i}+\sum_{j=1}^{s} b_{j} F_{j}^{\prime},
$$

it follows that $\left(p^{e_{4}}-1\right) b_{j} F_{j}^{\prime}$ is Cartier around $z$ for some $e_{4} \in \mathbb{Z}_{>0}$. Since $m \in\left\{m_{z}, 2 m_{z}\right\}$, we have that $2\left(p^{e_{4}}-1\right) b_{j} F_{j}^{\prime}$ is Cartier. Since char $k \neq 2$, we can find $e_{2} \in \mathbb{Z}_{>0}$ such that $\left(p^{e_{2}}-1\right) b_{j} F_{j}^{\prime}$ is Cartier. Thus, the claim follows.

\subsection{Flat families of log pairs and liftability.}

Definition 2.13. Given a reduced noetherian separated scheme $T$, a flat family of log pairs $(\mathcal{X}, \mathcal{B})$ over $T$ consists of the data of

- a normal scheme $\mathcal{X}$ and an effective $\mathbb{Q}$-divisor $\mathcal{B}$ on $\mathcal{X}$ such that $K_{\mathcal{X}}+\mathcal{B}$ is $\mathbb{Q}$-Cartier,

- a separated and flat morphism of finite type $\pi: \mathcal{X} \rightarrow T$ such that all the fibres of $\pi$ are geometrically normal, and

- for each irreducible component $\mathcal{B}_{i}$ of $\mathcal{B}$, the induced morphism $\left.\pi\right|_{\mathcal{B}_{i}}: \mathcal{B}_{i} \rightarrow T$ is flat and all the fibres of $\left.\pi\right|_{\mathcal{B}_{i}}$ are geometrically integral.

Definition 2.14. Let $(\mathcal{X}, \mathcal{B})$ be a flat family of log pairs over a reduced noetherian separated scheme $T$ and let $(X, B)$ be a log pair over an algebraically closed field $k$. We say that $(X, B)$ is a geometric fibre of $(\mathcal{X}, \mathcal{B})$, if there exists a cartesian diagram

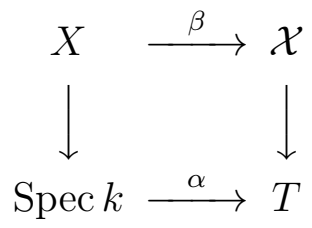

such that $\beta^{*}(\mathcal{B})=B$, where if we write $\mathcal{B}=\sum_{i=1}^{r} b_{i} \mathcal{B}_{i}$ with $\mathcal{B}_{1}, \ldots, \mathcal{B}_{r}$ prime components, then we define

$$
\beta^{*}(\mathcal{B}):=\sum_{i=1}^{r} b_{i} \beta^{-1}\left(\mathcal{B}_{i}\right) .
$$

Note that, for each $i=1, \ldots, r$, the scheme-theoretic inverse image $\beta^{-1}\left(\mathcal{B}_{i}\right)$ is a prime divisor on $X$. 
Definition 2.15. Let $X$ be a smooth variety over a perfect field $k$ of characteristic $p>0$, and let $D$ be a simple normal crossing divisor on $X$. Write $D=\sum_{i=1}^{r} D_{i}$, where $D_{i}$ are the irreducible components of $D$. We say that the pair $(X, D)$ lifts to characteristic zero over a smooth base if there exist

- a scheme $T$ smooth and separated over $\operatorname{Spec} \mathbb{Z}$,

- a smooth and separated morphism $\mathcal{X} \rightarrow T$,

- effective Cartier divisors $\mathcal{D}_{1}, \ldots, \mathcal{D}_{r}$ on $\mathcal{X}$ such that the schemetheoretic intersection $\bigcap_{i \in J} \mathcal{D}_{i}$ for any subset $J \subseteq\{1, \ldots, r\}$ is smooth over $T$, and

- a morphism $\alpha$ : Spec $k \rightarrow T$,

such that the base changes of the schemes $\mathcal{X}, \mathcal{D}_{1}, \ldots, \mathcal{D}_{r}$ over $T$ by $\alpha$ : Spec $k \rightarrow T$ are isomorphic to $X, D_{1}, \ldots, D_{r}$, respectively.

We refer to [EV92, Definition 8.11] for the definition of liftability to the second Witt vectors $W_{2}(k)$.

Remark 2.16. Under the same assumption as in Definition 2.15, if $(X, D)$ lifts to characteristic zero over a smooth base, then $(X, D)$ also lifts to $W_{2}(k)$. Indeed, since $W_{2}(k)$ is a henselian local ring [Fu15, Proposition 2.8.4], and $T$ is smooth over $\operatorname{Spec} \mathbb{Z}$, the morphism Spec $k \rightarrow T$ lifts to a morphism Spec $W_{2}(k) \rightarrow T$ by [Fu15, Proposition 2.8.13]:

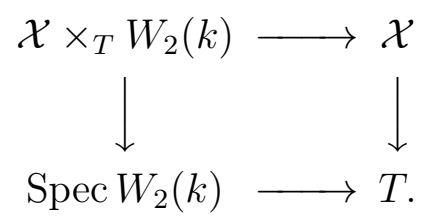

\section{Proof of the main theorem for $\epsilon$-Klt log del Pezzo}

The goal of this section is to prove the main theorem for $\epsilon$-klt log del Pezzo pairs, for any fixed $\epsilon>0$ (Proposition 3.2). The idea of the proof is that the family of $\epsilon$-klt $\log$ del Pezzos over Spec $\mathbb{Z}$ is bounded (Lemma 3.1). The same result is known to hold over a fixed algebraically closed field [Ale94, Theorem 6.9]. Our argument follows the same methods, however we include the proof for completeness.

Let $S$ be a noetherian separated scheme, let $Y$ be a scheme which is projective and flat over $S$ and let $H$ be an invertible sheaf on $Y$, which is ample over $S$. Let $\phi \in \mathbb{Q}[t]$ be an arbitrary polynomial. We define $\operatorname{Hilb}_{Y / S}^{\phi, H}$ to be the Hibert functor such that, for any scheme $T$ over $S$, $\operatorname{Hilb}_{Y / S}^{\phi, H}(T)$ is the set of closed subschemes in $Y \times_{S} T$ which are flat over $T$, and with Hilbert polynomial equal to $\phi$ with respect to the pull-back of $H$ on $Y \times_{S} T$ (see $\left[\mathrm{FGI}^{+} 05\right.$, Section 5.1] for more 
details). We also define $\operatorname{Div}_{Y / S}^{\phi, H}$ to be the functor such that, for any scheme $T$ over $S, \operatorname{Div}_{Y / S}^{\phi, H}(T)$ is the set of effective Cartier divisors on $Y \times{ }_{S} T$ which are flat over $T$, and with Hilbert polynomial equal to $\phi$ with respect to the pull-back of $H$ on $Y \times{ }_{S} T$ (see [ibid, Section 9.3]).

Since $\operatorname{Hilb}_{Y / S}^{\phi, H}$ is representable by a projective scheme over $S$ [ibid, Theorem 5.14 and Subsection 5.1.3], it follows that $\operatorname{Div}_{Y / S}^{\phi, H}$ is representable by a quasi-projective scheme over $S$ [ibid, Theorem 9.3.7].

Lemma 3.1. Let $I \subseteq(0,1) \cap \mathbb{Q}$ be a finite set and let $\epsilon>0$.

Then there exists a flat family of $\log$ pairs $(\mathcal{X}, \mathcal{B})$ over a reduced quasi-projective scheme $T$ over Spec $\mathbb{Z}$, such that every $\epsilon$-klt log del Pezzo pair $(X, B)$ over any algebraically closed field $k$ of characteristic $p>5$, with the coefficients of $B$ contained in $I$, is a geometric fibre of $(\mathcal{X}, \mathcal{B})$.

Proof. There exist positive integers $b$ and $n$ which satisfy the following property (e.g. see [Wit15, Corollary 1.4 and Remark 6.3]): for every $(X, B)$ over $k$ as in the Lemma, we can find a very ample divisor $H$ on $X$ such that

- $|H|$ embeds $X$ into $\mathbb{P}_{k}^{b}$,

- $n B$ is Cartier,

- $\left|\phi_{X, i}\right| \leq b$ for all $i \geq 0$ where $\phi_{X}=\sum_{i>0} \phi_{X, i} t^{i} \in \mathbb{Q}[t]$ is the Hilbert polynomial of $X$ with respect to $H$, and

- $\left|\psi_{n B, i}\right| \leq b$ for all $i \geq 0$ where $\psi_{n B}=\psi_{n B, 1} t+\psi_{n B, 0} \in \mathbb{Z}[t]$ is the Hilbert polynomial of $n B$ with respect to $H$.

We may assume that $I=(0,1) \cap \frac{1}{n} \mathbb{Z}$. Further, since $\operatorname{dim} X=2$, we have that $2 \phi_{X} \in \mathbb{Z}[t]$ and $\operatorname{deg} \phi_{X}=2$.

In particular, if $G$ is the functor defined by

$$
G:=\bigcup_{\phi} \operatorname{Hilb}_{\mathbb{P}_{\mathbb{Z}}^{b} / \mathbb{Z}}^{\phi, \mathcal{O}_{\mathbb{Z}}^{b} / \mathbb{Z}}
$$

where the union is taken over all $\phi=\sum \phi_{i} t^{i} \in \mathbb{Q}[t]$ such that $2 \phi \in \mathbb{Z}[t]$, $\operatorname{deg} \phi=2$, and $\left|\phi_{i}\right| \leq b$ for all $i \geq 0$, then $X \in G(\operatorname{Spec} k)$.

By $\mathrm{FGI}^{+} 05$, Theorem 5.14 and Subsection 5.1.3], the functor $G$ is representable by a projective scheme $S$ over Spec $\mathbb{Z}$. Let $\mathcal{U} \subseteq \mathbb{P}_{\mathbb{Z}}^{b} \times_{\mathbb{Z}} S$ be its universal closed subscheme. Let $\mathcal{H}$ be the pull-back of $\mathcal{O}_{\mathbb{P}_{\mathbb{Z}}^{b} / \mathbb{Z}}(1)$ to $\mathcal{U}$. Then $\mathcal{H}$ is ample over $S$. We have

$$
(n B \subseteq X) \in \bigcup_{\psi} \operatorname{Div}_{\mathcal{U} / S}^{\psi, \mathcal{H}}(\operatorname{Spec} k),
$$

where the union is taken over all $\psi=\psi_{1} t+\psi_{0} \in \mathbb{Z}[t]$ such that $\left|\psi_{0}\right| \leq b$ and $\left|\psi_{1}\right| \leq b$. 
Let $T$ be the reduction of the scheme representing $\bigcup_{\psi} \operatorname{Div}_{\mathcal{U} / S}^{\psi, \mathcal{H}}$. Note that $T$ is quasi-projective over Spec $\mathbb{Z}$. Let $\mathcal{B}^{\prime}$ be the universal effective Cartier divisor on $\mathcal{X}:=\mathcal{U} \times{ }_{S} T$. Let $\mathcal{B}:=\frac{1}{n} \mathcal{B}^{\prime}$. Take a generic point $\zeta$ of $T$. By taking a base change of some finite morphism of an open subset of $T$, we may assume that the fibres of the irreducible components of $\mathcal{B}$ over $\zeta$ are geometrically integral. Thus, there exists an open subscheme $U \subseteq T$, such that $\left(\left.\mathcal{X}\right|_{U},\left.\mathcal{B}\right|_{U}\right)$ is a flat family of log pairs. By replacing $T$ by $U \amalg T \backslash U$ and repeating the same argument to $T \backslash U$, we may conclude the proof.

We now show the main result of this Section.

Proposition 3.2. Let $I \subseteq(0,1) \cap \mathbb{Q}$ be a finite set and let $\epsilon>0$. Then, there exists a positive integer $p(I, \epsilon)$ which satisfies the following property:

Let $(X, B)$ be an $\epsilon$-klt log del Pezzo pair over an algebraically closed field of characteristic $p>p(I, \epsilon)$ such that the coefficients of $B$ are contained in $I$. Then there exists a log resolution $\mu: V \rightarrow X$ of $(X, B)$ such that $\left(V, \operatorname{Exc}(\mu) \cup \mu_{*}^{-1}(\operatorname{Supp} B)\right)$ lifts to characteristic zero over a smooth base.

Proof. We fix a positive integer $p(I, \epsilon)>5$. We will replace $p(I, \epsilon)$ by a larger number if necessary. By Lemma 3.1, there exists a flat family of $\log$ pairs $(\mathcal{X}, \mathcal{B})$ over a reduced quasi-projective scheme $T$ over Spec $\mathbb{Z}$, such that every pair $(X, B)$ as in the Proposition is a geometric fibre of the pair $(\mathcal{X}, \mathcal{B})$.

Claim: Let $T^{\prime}$ be an irreducible component of $T$ such that the field $K\left(T^{\prime}\right)$ is of characteristic zero.

Then there exists a dominant morphism $S \rightarrow T^{\prime}$ from an integral scheme $S$ which satisfies the following properties:

(a) $S$ is smooth over Spec $\mathbb{Z}$.

(b) If we set $\mathcal{X}_{S}:=\mathcal{X} \times_{T} S$, then there exists a projective birational morphism $\mu_{S}: \mathcal{V}_{S} \rightarrow \mathcal{X}_{S}$ over $S$ such that the induced morphism $\mu_{S, s}: \mathcal{V}_{s} \rightarrow \mathcal{X}_{s}$ between any fibres over $s \in S$ is birational, $\mathcal{V}_{S}$ is smooth over $S$, and

$$
\operatorname{Exc}\left(\mu_{S}\right) \cup\left(\mu_{S}\right)_{*}^{-1}(\operatorname{Supp} \mathcal{B})
$$

is simple normal crossing over $S$, i.e. all the strata are smooth over $S$. 
We first show the Proposition, assuming the Claim. By noetherian induction, we can find a surjective morphism

$$
\left(\coprod_{1 \leq i \leq \alpha} S_{i}\right) \amalg\left(\coprod_{1 \leq j \leq \beta} S_{j}^{\prime}\right) \rightarrow T
$$

such that all $S_{i}$ and $S_{j}^{\prime}$ are separated integral schemes of finite type over Spec $\mathbb{Z}$, any $K\left(S_{i}\right)$ (resp. $K\left(S_{j}^{\prime}\right)$ ) is of characteristic zero (resp. $p_{j}>0$ ), and each $S_{i}$ satisfies properties (a) and (b). Then the Proposition follows after possibly increasing $p(I, \epsilon)$ so that $p(I, \epsilon) \geq p_{j}$ for all $j=$ $1, \ldots, \beta$.

We now show the Claim. We set $S:=T^{\prime}$. It is enough to show that properties (a) and (b) hold after possibly replacing $S$ by a finite cover (i.e. a finite surjective morphism from an integral scheme) of an open subset.

By our assumption, the field $K(S)=\mathcal{O}_{S, \xi}$ is of characteristic zero, where $\xi$ is the generic point of $S$. Thus, after replacing $S$ by an open subset, we may assume that $S$ is smooth over Spec $\mathbb{Z}$, hence (a) holds.

Let $\left(X_{\bar{\xi}}, B_{\bar{\xi}}\right)$ be the base change to the algebraic closure $\bar{\xi}$ of the generic fibre $\left(X_{\xi}, B_{\xi}\right)$ and take a log resolution $\mu_{\bar{\xi}}: V_{\bar{\xi}} \rightarrow X_{\bar{\xi}}$ of $\left(X_{\bar{\xi}}, B_{\bar{\xi}}\right)$. After replacing $S$ by a finite cover of an open subset, we may assume that there exists a projective birational morphism over $S$

$$
\mu_{S}: \mathcal{V}_{S} \rightarrow \mathcal{X}_{S}
$$

whose base change to $\bar{\xi}$ is the same as $V_{\bar{\xi}} \rightarrow X_{\bar{\xi}}$, where $\mathcal{X}_{S}:=\mathcal{X} \times_{T} S$.

We check that property (b) holds, after replacing $S$ by an open subset. Indeed, since $\mu_{\bar{\xi}}: V_{\bar{\xi}} \rightarrow X_{\bar{\xi}}$ is a projective birational morphism of surfaces, so are the morphisms $\mathcal{V}_{S, s} \rightarrow \mathcal{X}_{S, s}$ between the geometric fibres, for any geometric point $s$ in an open neighbourhood of $\xi$. Moreover, $V_{\bar{\xi}}$ and any stratum of $\operatorname{Exc}\left(\mu_{\bar{\xi}}\right) \cup\left(\mu_{\bar{\xi}}\right)_{*}^{-1}\left(\operatorname{Supp} \mathcal{B}_{\bar{\xi}}\right)$ are smooth, hence $\mathcal{V}_{S}$ and all the strata of $\operatorname{Exc}\left(\mu_{S}\right) \cup\left(\mu_{S}\right)_{*}^{-1}(\operatorname{Supp} \mathcal{B})$ are smooth.

Remark 3.3. By Proposition 3.2, it follows that $\epsilon$-klt log del Pezzo pairs in large characteristic satisfy (2) of Theorem 1.1. By [SS10, Theorem 1.2] and noetherian induction, it follows that (1) of Theorem 1.1 holds for $\epsilon$-klt log del Pezzo pairs in large characteristic. However, we do not use this fact in this paper.

\section{Liftability TO CHARACTERISTIC ZERO}

The goal of this section is to study plt pairs $\left(Z, C_{Z}+B_{Z}\right)$, such that $\left\llcorner C_{Z}+B_{Z}\right\lrcorner=C_{Z}$ is a prime divisor and $K_{Z}+C_{Z}+B_{Z} \equiv 0$. These pairs appear in the proof of Theorem 1.1, after running a suitable MMP, 
starting from some model over a log del Pezzo pair $(X, B)$ (cf. Section 5).

We first show that, in large characteristic, such a pair $\left(Z, C_{Z}+\right.$ $B_{Z}$ ) admits a log resolution which lifts to characteristic zero (Proposition 4.2). Then, in order to show that also the pair $(X, B)$ admits a log resolution which lifts to characteristic zero, we study the behaviour of such a liftability property under blow-ups (Lemma 4.3).

The following result is a consequence of Lemma 3.1;

Lemma 4.1. Let $I \subseteq(0,1) \cap \mathbb{Q}$ be a DCC set.

Then there exists a flat family of $\log$ pairs $(\mathcal{X}, \mathcal{C}+\mathcal{B})$ over a reduced quasi-projective scheme $T$ over Spec $\mathbb{Z}$, such that any two dimensional projective plt pair $(X, C+B)$, over any algebraically closed field $k$ of characteristic $p>5$, satisfying

- $\lfloor C+B\rfloor=C$,

- the coefficients of $B$ are contained in $I$,

- $K_{X}+C+B \equiv 0$, and

- $C$ is ample,

is a geometric fibre of $(\mathcal{X}, \mathcal{C}+\mathcal{B})$.

Proof. As in the proof of Proposition 2.5, there exists a positive integer $m$ depending only on $I$ such that

$$
m\left(K_{X}+C+B\right) \sim 0 \quad \text { and } \quad m C \text { is Cartier }
$$

for any pair $(X, C+B)$ satisfying the assumptions in the Lemma.

In particular, $2 m\left(K_{X}+\frac{1}{2} C+B\right)$ is Cartier, and if $\epsilon \in\left(0, \frac{1}{2 m}\right)$ then $\left(X, \frac{1}{2} C+B\right)$ is $\epsilon$-klt. Thus, Lemma 3.1 implies that there exists a flat family $\left(\mathcal{X}, \frac{1}{2} \mathcal{C}+\mathcal{B}\right)$ of $\log$ pairs such that $\left(X, \frac{1}{2} C+B\right)$ is a geometric fibre of $\left(\mathcal{X}, \frac{1}{2} \mathcal{C}+\mathcal{B}\right)$. In particular, $(\mathcal{X}, \mathcal{C}+\mathcal{B})$ is the required family.

Proposition 4.2. Let $I \subseteq(0,1) \cap \mathbb{Q}$ be a finite set.

Then there exists a positive integer $p(I)$ which satisfies the following property:

For any two dimensional projective plt pair $(Z, C+B)$ over an algebraically closed field of characteristic $p>p(I)$ such that

- $\llcorner C+B\lrcorner=C$,

- $C$ is ample,

- $K_{Z}+C+B \equiv 0$, and

- the coefficients of $B$ are contained in $I$,

there exists a birational morphism $\pi: W \rightarrow Z$ such that

- $W$ is a smooth projective surface and $\operatorname{Supp}\left(\pi_{*}^{-1}(C+B)\right) \cup \operatorname{Exc}(\pi)$ is simple normal crossing, and 
- $\left(W, \operatorname{Supp}\left(\pi_{*}^{-1}(C+B)\right) \cup \operatorname{Exc}(\pi)\right)$ lifts to characteristic zero over a smooth base.

Proof. We can apply the same argument as in the proof of Proposition 3.2 after replacing $(X, B)$ (resp. Lemma 3.1) by $(Z, C+B)$ (resp. Lemma 4.1).

Lemma 4.3. Let $Z$ be a smooth projective surface over an algebraically closed field $k$ of characteristic $p>0$ and let $D$ be a reduced simple normal crossing divisor on $Z$. Let $\pi: Z^{\prime} \rightarrow Z$ be the blow up at a point $q$ contained in the singular locus of $D$ and let $E$ be the $\pi$-exceptional $(-1)$-curve. If $(Z, D)$ lifts to characteristic zero over a smooth base, then so does $\left(Z^{\prime}, \pi_{*}^{-1} D+E\right)$.

Proof. We may write $D=\sum_{i=1}^{n} D_{i}$, where $D_{1}, \ldots, D_{n}$ are the irreducible components. Since $(Z, D)$ lifts to characteristic zero, there exists a pair $\left(\mathcal{Z}, \mathcal{D}=\sum_{i=1}^{n} \mathcal{D}_{i}\right)$ over a smooth separated scheme $T$ over Spec $\mathbb{Z}$ and a morphism $\alpha$ : Spec $k \rightarrow T$, such that the base changes of the schemes $\mathcal{X}, \mathcal{D}_{1}, \ldots, \mathcal{D}_{n}$ over $T$ by $\alpha$ : Spec $k \rightarrow T$ are isomorphic to $X, D_{1}, \ldots, D_{n}$ respectively and satisfy the same properties as in Definition 2.15.

By assumption, there exist unique $D_{i}, D_{j}$ such that $q \in D_{i} \cap D_{j}$. Let $\mathcal{C}$ be the irreducible component of $\mathcal{D}_{i} \cap \mathcal{D}_{j}$ such that $q \in \mathcal{C}$. Let $\bar{\pi}: \mathcal{Z}^{\prime} \rightarrow \mathcal{Z}$ be the blow-up along $\mathcal{C}$. Then, $\mathcal{Z}^{\prime}$ is a lift of $Z^{\prime}$. Indeed, since $\mathcal{D}_{i} \cap \mathcal{D}_{j}$ is smooth over $T$, so is $\mathcal{C}$. It follows that $\mathcal{Z}^{\prime}$ is smooth over $T$ and has connected fibres over $T$ (e.g. see the proof of [Liu02, Section 8, Theorem 1.19]). By [ibid], the exceptional divisor $\mathcal{E}$ of $\bar{\pi}$ is a $\mathbb{P}^{1}$-bundle over $\mathcal{C}$. Thus, all the assumptions in Definition 2.15 hold true for the pairs $\left(Z^{\prime}, \pi_{*}^{-1} D+E\right)$ and $\left(\mathcal{Z}^{\prime}, \bar{\pi}_{*}^{-1} \mathcal{D}+\mathcal{E}\right)$ over $T$.

Remark 4.4. By Lemma 4.3, we may assume that the log resolution appearing in (2) of Theorem 1.1 factors through the terminalisation $\eta: W \rightarrow X$ of $(X, B)$, i.e. if we write

$$
K_{W}+B_{W}=\eta^{*}\left(K_{X}+B\right),
$$

then $\left(W, B_{W}\right)$ is terminal.

\section{Non- $\epsilon$-KLt CASE AND PRoOf of Theorem 1.1}

In Section 3, we showed that $\epsilon$-klt log del Pezzo surfaces form a bounded family. The goal of this section is to study log del Pezzo surfaces $(X, B)$ over an algebraically closed field $k$, which are not $\epsilon$-klt for $0<\epsilon \ll 1$. In particular, this yields a proof of our main Theorem (Theorem 1.1). 
Our method consists of constructing a log canonical pair $\left(Z, C_{Z}+B_{Z}\right)$ from $(X, B)$, such that $\left\lfloor C_{Z}+B_{Z}\right\rfloor=C_{Z}$ is prime and $-\left(K_{Z}+C_{Z}+B_{Z}\right)$ is nef. If $K_{Z}+C_{Z}+B_{Z}$ is not numerically trivial, then, by using global $F$-adjunction (see Subsection 2.2), we show that $\left(Z, C_{Z}+B_{Z}\right)$ is globally $F$-split, provided that the characteristic of $k$ is large enough. This in turn implies that $(X, B)$ is globally $F$-regular.

Unfortunately, if $K_{Z}+C_{Z}+B_{Z}$ is numerically trivial, then $\left(Z, C_{Z}+\right.$ $B_{Z}$ ) does not need to be globally $F$-split [CTW16, Theorem 1.1]. Thus, we need to consider two different cases, depending on whether $\left(Z, C_{Z}+\right.$ $\left.B_{Z}\right)$ is plt or not. If the pair is plt (resp. non-plt), then we show that condition (2) (resp. condition (1)) of Theorem 1.1 holds.

Proposition 5.1. Let $I \subseteq(0,1) \cap \mathbb{Q}$ be a finite set.

Then there exists a rational number $\epsilon(I)>0$ which satisfies the following property:

Let $(X, B)$ be a two dimensional projective klt pair over an algebraically closed field such that

- $-\left(K_{X}+B\right)$ is ample,

- $(X, B)$ is not $\epsilon(I)-k l t$, and

- the coefficients of $B$ are contained in $I$.

Then there exist birational morphisms

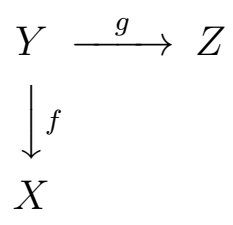

of projective klt surfaces such that if we denote $C=\operatorname{Exc}(f), C_{Z}=g_{*} C$, $B_{Y}=f_{*}^{-1} B$ and $B_{Z}=g_{*} B_{Y}$, then $C$ is a prime divisor, $C_{Z} \neq 0$,

(1) if $a=a(C, X, B)$ so that

$$
K_{Y}+(1-a) C+B_{Y}=f^{*}\left(K_{X}+B\right),
$$

then $a \in(0, \epsilon(I)]$,

(2) there exists a $\mathbb{Q}$-divisor $B_{Y}^{+} \geq B_{Y}$ such that

$$
K_{Y}+C+B_{Y}^{+}=g^{*}\left(K_{Z}+C_{Z}+B_{Z}\right)
$$

and

$$
\operatorname{Exc}(g)=\operatorname{Supp}\left(B_{Y}^{+}-B_{Y}\right),
$$

(3) $-\left(K_{Z}+C_{Z}+B_{Z}\right)$ is nef,

(4) $\left(Z, C_{Z}+B_{Z}\right)$ is log canonical, and

(5) $\left(Z, b C_{Z}+B_{Z}\right)$ is klt and $-\left(K_{Z}+b C_{Z}+B_{Z}\right)$ is ample for some rational number $b \in(1-a, 1)$. 
Proof. Pick any rational number $\epsilon(I) \in(0,1)$ such that $\epsilon<1-c$ for any $c \in I$. We will replace $\epsilon(I)$ by a smaller number, if necessary.

Let $(X, B)$ be a log pair which satisfies the assumptions in the Proposition. Let $C$ be an exceptional divisorial valuation such that $a:=a(C, X, B)$ is minimal and let $f: Y \rightarrow X$ be a projective birational morphism such that $\operatorname{Exc}(f)=C$. By assumption, $a \in(0, \epsilon(I)]$. Thus, (1) holds.

Since $-\left(K_{X}+B\right)$ is ample, we can find a rational number $b \in(1-a, 1)$ such that $\left(Y, b C+B_{Y}\right)$ is klt, and $-\left(K_{Y}+b C+B_{Y}\right)$ is ample. We run a $-\left(K_{Y}+C+B_{Y}\right)-\mathrm{MMP}$ with scaling of $C$ :

$$
\begin{aligned}
\left(Y, C+B_{Y}\right)=:\left(Y_{0}, C_{0}+B_{0}\right) \stackrel{g_{0}}{\longrightarrow}\left(Y_{1}, C_{1}+B_{1}\right) \stackrel{g_{1}}{\longrightarrow} \cdots \\
\cdots \stackrel{g_{n-1}}{\longrightarrow}\left(Y_{n}, C_{n}+B_{n}\right)=:\left(Z, C_{Z}+B_{Z}\right),
\end{aligned}
$$

where $C_{i}:=\left(g_{i-1}\right)_{*} C_{i-1}$ and $B_{i}:=\left(g_{i-1}\right)_{*} B_{i-1}$.

By definition of the MMP with scaling, we get a sequence of rational numbers

$$
b<b_{0} \leq b_{1} \leq \cdots \leq b_{n-1}
$$

such that

$$
b_{i}:=\max \left\{t>b \mid-\left(K_{Y_{i}}+t C_{i}+B_{i}\right) \text { is nef }\right\}
$$

and $g_{i}: Y_{i} \rightarrow Y_{i+1}$ is a birational morphism, such that $\rho\left(Y_{i} / Y_{i+1}\right)=1$ and

$$
K_{Y_{i}}+b_{i} C_{i}+B_{i}=g_{i}^{*}\left(K_{Y_{i+1}}+b_{i} C_{i+1}+B_{i+1}\right) .
$$

Since $-\left(K_{Y_{i}}+b C_{i}+B_{i}\right)$ is ample for any $i=0, \ldots, n$, each step of the MMP is $\left(K_{Y_{i}}+b C_{i}+B_{i}\right)$-negative, and in particular $\left(Y_{i}, b C_{i}+B_{i}\right)$ is klt. Thus, (5) holds.

Since the coefficients of $B_{Z}$ belong to the finite set $I$ and $\left(Z, b C_{Z}+\right.$ $B_{Z}$ ) is klt, by ACC for the log canonical threshold in dimension two Ale93, Theorem 1.1], after possibly replacing $\epsilon(I)$ by a smaller value depending only on $I$, we may assume that the pair $\left(Z, C_{Z}+B_{Z}\right)$ is log canonical, hence (4) holds.

Since $C_{i}$ is $g_{i}$-ample for all $i=0, \ldots, n-1$, it follows that $C_{Z} \neq 0$. We may write

$$
g^{*}\left(K_{Z}+C_{Z}+B_{Z}\right)=K_{Y}+C+B_{Y}+R
$$

for some $g$-exceptional $\mathbb{Q}$-divisor $R$ on $Y$. Since the MMP is $-\left(K_{Y}+\right.$ $C+B_{Y}$ )-negative, it follows that $R \geq 0$ and the support of $R$ coincides with the exceptional locus of $g$. In particular, if $B_{Y}^{+}=B_{Y}+R$, then (2) holds. 
Thus, it is enough to show (3). We assume by contradiction that $-\left(K_{Z}+C_{Z}+B_{Z}\right)$ is not nef. Thus, there exists a $-\left(K_{Z}+C_{Z}+B_{Z}\right)$ negative Mori fibre space $\pi: Z \rightarrow T$ and, in particular,

$$
\left(K_{Z}+C_{Z}+B_{Z}\right) \cdot F>0
$$

for any curve $F$ contained in a fibre of $\pi$. On the other hand, since $-\left(K_{Z}+b C_{Z}+B_{Z}\right)$ is ample, it follows that

$$
\left(K_{Z}+b C_{Z}+B_{Z}\right) \cdot F<0 .
$$

Thus, $C_{Z}$ is $\pi$-horizontal and we can find a rational number $b^{\prime} \in(b, 1)$ such that

$$
K_{Z}+b^{\prime} C_{Z}+B_{Z} \equiv_{\pi} 0 .
$$

Since $1-\epsilon(I)<b<b^{\prime}<1$, after possibly replacing $\epsilon(I)$ by a smaller number depending only on $I$, we get a contradiction by Lemma 2.3. Thus, (3) holds.

Before we proceed with the proof of Theorem 1.1, we recall the following criterion for global $F$-regularity:

Lemma 5.2. Let $(Z, C+B)$ be a log pair, where $\lfloor C+B\rfloor=C$ is a reduced divisor. Let $H$ be an effective $\mathbb{Q}$-divisor on $Z$ such that $Z \backslash \operatorname{Supp}(H+C)$ is affine. Assume that

(1) $(Z, B)$ is strongly $F$-regular, and

(2) there exists $\delta>0$ such that $(Z, C+B+\delta H)$ is globally $F$-split.

Then $(Z, \lambda C+B)$ is globally $F$-regular for every $\lambda \in[0,1)$.

Proof. This follows from [SS10, Theorem 3.9].

We now prove Theorem 1.1.

Proof of Theorem 1.1. Let $\epsilon(I)>0$ be the positive rational number as in Proposition 5.1 and let $p(I):=p(I, \epsilon(I))$ be the positive integer as in Proposition 3.2. We will replace $\epsilon(I)$ (resp. $p(I)$ ) by a smaller (resp. larger) value if necessary.

Let $(X, B)$ be a log del Pezzo pair over an algebraically closed field of characteristic $p>p(I)$. If $(X, B)$ is $\epsilon(I)$-klt, then Proposition 3.2 implies (2) of Theorem 1.1. Thus, we may assume that $(X, B)$ is not $\epsilon(I)-\mathrm{klt}$. By Proposition 5.1, there exist birational morphisms

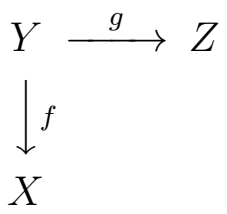

which satisfy the properties of Proposition 5.1. 
Claim: After possibly replacing $p(I)$ by a larger value depending only on $I$, the following hold:

(a) $\left(p^{e}-1\right) B_{Z}$ is a $\mathbb{Z}$-divisor for some $e \in \mathbb{Z}_{>0}$,

(b) $\left(Z, C_{Z}+B_{Z}\right)$ is sharply $F$-pure and $\left(Z, \lambda C_{Z}+B_{Z}\right)$ is strongly $F$-regular for any $\lambda \in[0,1)$, and

(c) if $\left(Z, \lambda C_{Z}+B_{Z}\right)$ is globally $F$-regular for any $\lambda \in[0,1)$, then $(X, B)$ is globally $F$-regular.

We now prove the Claim. (a) is clear. (4) and (5) of Proposition 5.1 imply that $\left(Z, \lambda C_{Z}+B_{Z}\right)$ is klt for any $\lambda \in[0,1)$. Thus, after possibly replacing $p(I)$ by a larger number depending only on $I$, by [CGS16, Theorem 1.1], we may assume that $\left(Z, \lambda C_{Z}+B_{Z}\right)$ is strongly $F$-regular. By [CTW16, Proposition 3.3], $\left(Z, C_{Z}+B_{Z}\right)$ is $F$-pure, hence it is sharply $F$-pure by (a). Thus (b) holds.

We now show (c). Assume that $\left(Z, \lambda C_{Z}+B_{Z}\right)$ is globally $F$-regular for any $\lambda \in(0,1)$. By (2) of Proposition [5.1, if $\lambda \in(0,1)$ is a rational number which is sufficiently close to 1 , then

$$
K_{Y}+\lambda C+B_{Y}^{\prime}=g^{*}\left(K_{Z}+\lambda C_{Z}+B_{Z}\right),
$$

for some $\mathbb{Q}$-divisor $B_{Y}^{\prime} \geq B_{Y}$. By [HX15, Proposition 2.11], it follows that $\left(Y, \lambda C+B_{Y}\right)$ is globally $F$-regular for some $\lambda \in(0,1)$. By [CTW16, Lemma 2.2], it follows that $(X, B)$ is globally $F$-regular as well. Thus, (c) holds and this completes the proof of the Claim.

By (3) of Proposition 5.1, it follows that $-\left(K_{Z}+C_{Z}+B_{Z}\right)$ is nef. By [Tan14, Theorem 1.2], it is semi-ample. In order to prove the Theorem, we consider the following four cases separately:

- $\kappa\left(Z,-\left(K_{Z}+C_{Z}+B_{Z}\right)\right)=2$,

- $\kappa\left(Z,-\left(K_{Z}+C_{Z}+B_{Z}\right)\right)=1$,

- $\kappa\left(Z,-\left(K_{Z}+C_{Z}+B_{Z}\right)\right)=0$ and $\left(Z, C_{Z}+B_{Z}\right)$ is not plt,

- $\kappa\left(Z,-\left(K_{Z}+C_{Z}+B_{Z}\right)\right)=0$ and $\left(Z, C_{Z}+B_{Z}\right)$ is plt.

If one of the first three cases (resp. if the last case) holds, then we will show that (1) (resp. (2)) of Theorem 1.1 holds. If $C_{Z}$ is normal, then we define $\left(C_{Z}, B_{C_{Z}}\right)$ by adjunction

$$
\left.\left(K_{Z}+C_{Z}+B_{Z}\right)\right|_{C_{Z}}=K_{C_{Z}}+B_{C_{Z}} .
$$

Case 1: $\kappa\left(Z,-\left(K_{Z}+C_{Z}+B_{Z}\right)\right)=2$.

Since $-\left(K_{Z}+C_{Z}+B_{Z}\right)$ is semi-ample, we can contract the curves $\Gamma$ with $\left(K_{Z}+C_{Z}+B_{Z}\right) \cdot \Gamma=0$. In this contraction, $C_{Z}$ is not contracted, because if $\left(K_{Z}+C_{Z}+B_{Z}\right) \cdot C_{Z}=0$, then $C_{Z}^{2}<0$, which contradicts

$$
(1-b) C_{Z}^{2}=\left(K_{Z}+C_{Z}+B_{Z}\right) \cdot{ }_{28} C_{Z}-\left(K_{Z}+b C_{Z}+B_{Z}\right) \cdot C_{Z}>0 .
$$


Thus, by [HX15, Proposition 2.11], we may assume that $-\left(K_{Z}+C_{Z}+\right.$ $\left.B_{Z}\right)$ is ample. By [Tan14, Theorem 3.19] and

$$
\left(K_{Z}+C_{Z}\right) \cdot C_{Z} \leq\left(K_{Z}+C_{Z}+B_{Z}\right) \cdot C_{Z}<0
$$

it follows that $C_{Z} \simeq \mathbb{P}^{1}$.

By Lemma 2.11, there exists an effective $\mathbb{Q}$-divisor $H$ such that $\left(Z, C_{Z}+B_{Z}+H\right)$ is $\log$ canonical and the Cartier index of $K_{Z}+$ $C_{Z}+B_{Z}+H$ is not divisible by $p$. After replacing $H$ by $H+H^{\prime}$ for some ample effective $\mathbb{Q}$-divisor $H^{\prime}$, we may assume that $Z \backslash \operatorname{Supp} H$ is affine. After replacing $H$ by a smaller multiple, we may assume that $-\left(K_{Z}+C_{Z}+B_{Z}+H\right)$ is ample.

We now show that $\left(C_{Z}, B_{C_{Z}}+\left.H\right|_{C_{Z}}\right)$ is globally $F$-split. If $\left(C_{Z}, B_{C_{Z}}+\right.$ $\left.\left.H\right|_{C_{Z}}\right)$ is not klt, then Lemma 2.7 implies that $\left(C_{Z}, B_{C_{Z}}+\left.H\right|_{C_{Z}}\right)$ is globally $F$-split. If $\left(C_{Z}, B_{C_{Z}}+\left.H\right|_{C_{Z}}\right)$ is klt, then by [CGS16, Corollary 4.1 , after possibly replacing $p(I)$ by a larger value depending only on $I$, we may assume that $\left(C_{Z}, B_{C_{Z}}\right)$ is globally $F$-regular. After possibly replacing $H$ by a smaller multiple again, we may assume that $\left(C_{Z}, B_{C_{Z}}+\left.H\right|_{C_{Z}}\right)$ is globally $F$-split.

By Lemma 2.7, it follows that $\left(Z, C_{Z}+B_{Z}+H\right)$ is globally $F$-split. Thus, (b) of the Claim and Lemma 5.2 imply that $\left(Z, \lambda C_{Z}+B_{Z}\right)$ is globally $F$-regular for any $\lambda \in[0,1)$. By (c) of the Claim, it follows that $(X, B)$ is globally $F$-regular.

Case 2: $\kappa\left(Z,-\left(K_{Z}+C_{Z}+B_{Z}\right)\right)=1$.

Since $-\left(K_{Z}+C_{Z}+B_{Z}\right)$ is semi-ample, there exist a morphism $\pi: Z \rightarrow$ $T$ onto a smooth projective curve $T$ and an ample $\mathbb{Q}$-divisor $A$ on $T$ such that $\pi_{*} \mathcal{O}_{Z}=\mathcal{O}_{T}$ and $-\left(K_{Z}+C_{Z}+B_{Z}\right) \equiv \pi^{*} A$. By (5) of Proposition [5.1 and by [HX15, Proposition 2.11], after running a $\left(K_{Z}+b C_{Z}+B_{Z}\right)$-MMP over $T$, we may assume that $\pi: Z \rightarrow T$ is a $\left(K_{Z}+b C_{Z}+B_{Z}\right)$-negative Mori fibre space. In particular, $C_{Z}$ is $\pi$-horizontal. By [Tan14, Theorem 3.19] and

$$
\left(K_{Z}+C_{Z}\right) \cdot C_{Z} \leq\left(K_{Z}+C_{Z}+B_{Z}\right) \cdot C_{Z}<0,
$$

it follows that $C_{Z} \simeq \mathbb{P}^{1}$.

By (a) of the Claim, we can apply Lemma 2.12, and there exists an effective $\mathbb{Q}$-divisor $E$, whose support is contained in some fibres of $\pi$, and such that $\left(Z, C_{Z}+B_{Z}+E\right)$ is log canonical and the Cartier index of $K_{Z}+C_{Z}+B_{Z}+E$ is not divisible by $p$. After possibly replacing $E$ by a smaller multiple, we may assume that $-\left(K_{Z}+C_{Z}+B_{Z}+E\right) \equiv \pi^{*} A^{\prime}$ for some ample $\mathbb{Q}$-divisor $A^{\prime}$ on $T$. In particular, $-\left(K_{C_{Z}}+B_{C_{Z}}+\left.E\right|_{C_{Z}}\right)$ is ample.

By the same argument as in Case $1,\left(C_{Z}, B_{C_{Z}}+\left.E\right|_{C_{Z}}\right)$ is globally $F$-split. Thus, by Lemma $2.8,\left(Z, C_{Z}+B_{Z}+E\right)$ is globally $F$-split. 
Again by the same argument as in Case 1 , it follows that $(X, B)$ is globally $F$-regular.

Case 3: $\kappa\left(Z,-\left(K_{Z}+C_{Z}+B_{Z}\right)\right)=0$ and $\left(Z, C_{Z}+B_{Z}\right)$ is not plt.

By Proposition [2.5, after possibly replacing $p(I)$ by a larger value depending only on $I$, we may assume that $\left(p^{e}-1\right)\left(K_{Z}+C_{Z}+B_{Z}\right)$ is Cartier for some positive integer $e$. Thus, Lemma 2.9 implies that $\left(Z, C_{Z}+B_{Z}\right)$ is globally $F$-split. By (5) of Proposition [5.1, it follows that $-\left(K_{Z}+b C_{Z}+B_{Z}\right)$ is ample, and in particular also $C_{Z}$ is ample. Thus, (b) of the Claim and Lemma 5.2 imply that $\left(Z, \lambda C_{Z}+B_{Z}\right)$ is globally $F$-regular for any $\lambda \in[0,1)$. By (c) of the Claim, it follows that $(X, B)$ is globally $F$-regular.

Case 4: $\kappa\left(Z,-\left(K_{Z}+C_{Z}+B_{Z}\right)\right)=0$ and $\left(Z, C_{Z}+B_{Z}\right)$ is plt.

By Proposition 4.2, after possibly replacing $p(I)$ by a larger number depending only on $I$, there exists a $\log$ resolution $\pi: W \rightarrow Z$ of $(Z, C+$ $B)$ such that

$$
\left(W, D_{W}:=\operatorname{Supp}\left(\pi_{*}^{-1}(C+B)\right) \cup \operatorname{Exc}(\pi)\right)
$$

lifts to characteristic zero over a smooth base. We may write

$$
K_{W}+D_{W}^{\prime}=\pi^{*}\left(K_{Z}+C_{Z}+B_{Z}\right) .
$$

for some $\mathbb{Q}$-divisor $D_{W}^{\prime}$ on $W$. Note that $\operatorname{Supp} D_{W}^{\prime} \subseteq \operatorname{Supp} D_{W}$. By (2) of Proposition 5.1, the birational morphism $g: Y \rightarrow Z$ only extracts prime divisors $E$ such that $a\left(E, W, D_{W}^{\prime}\right)=a\left(E, Z, C_{Z}+B_{Z}\right)<1$. Thus, there exists a sequence of blow-ups

$$
\varphi: V:=W_{m} \stackrel{\varphi_{m-1}}{\longrightarrow} \cdots \stackrel{\varphi_{1}}{\longrightarrow} W_{1} \stackrel{\varphi_{0}}{\longrightarrow} W_{0}:=W
$$

which satisfies the following properties:

- We define a divisor $D_{i}$ on $W_{i}$ inductively by

$$
\begin{aligned}
D_{0} & :=D_{W} \\
K_{W_{i+1}}+D_{i+1} & =\varphi_{i}^{*}\left(K_{W_{i}}+D_{i}\right) .
\end{aligned}
$$

- Each $\varphi_{i}$ is the blow-up of a point contained in $\operatorname{Sing}\left(\operatorname{Supp} D_{i}\right)$.

- The composite arrow $\psi: V \stackrel{\varphi}{\rightarrow} W \stackrel{\pi}{\rightarrow} Z$ factors through $Y$.

Note that each $D_{i}$ is a reduced simple normal crossing divisor. Therefore, by Lemma 4.3, $\left(V, \psi_{*}^{-1}\left(C_{Z}+B_{Z}\right) \cup \operatorname{Exc}(\psi)\right)$ lifts to characteristic zero over a smooth base. Thus, (2) of the Theorem holds.

\section{Kawamata-Viehweg vanishing for log del Pezzo SURFACES}

The goal of this section is to prove Theorem 1.2. We begin with the following: 
Lemma 6.1. Assume that char $k>2$. Let $(X, \Delta)$ be a two dimensional projective klt pair. Suppose that there exists a log resolution $\mu: V \rightarrow X$ of $(X, \Delta)$ such that

$$
\left(V, \operatorname{Exc}(\mu) \cup \mu_{*}^{-1}(\operatorname{Supp} \Delta)\right)
$$

lifts to $W_{2}(k)$. Let $D$ be a $\mathbb{Z}$-divisor on $X$ such that $D-\left(K_{X}+\Delta\right)$ is ample.

Then,

$$
H^{i}\left(X, \mathcal{O}_{X}(D)\right)=0 \quad \text { for any } i>0
$$

Proof. Let

$$
M=K_{V}+\left\ulcorner\mu^{*}\left(D-\left(K_{X}+\Delta\right)\right)\right\urcorner .
$$

We may find a $\mu$-exceptional $\mathbb{Q}$-divisor $E \geq 0$ such that

- $M=K_{V}+\left\ulcorner\mu^{*}\left(D-\left(K_{X}+\Delta\right)\right)-E\right\urcorner$, and

- $\mu^{*}\left(D-\left(K_{X}+\Delta\right)\right)-E$ is ample.

Note that $\left\{\mu^{*}\left(D-\left(K_{X}+\Delta\right)\right)-E\right\}$ is simple normal crossing and lifts to $W_{2}(k)$. Since char $k>2$, [Har98, Corollary 3.8] implies that

$$
H^{i}\left(V, \mathcal{O}_{V}(M)\right)=0
$$

for every $i>0$. Consider the Leray spectral sequence:

$$
E_{2}^{i, j}:=H^{i}\left(X, R^{j} \mu_{*} \mathcal{O}_{V}(M)\right) \Rightarrow H^{i+j}\left(V, \mathcal{O}_{V}(M)\right)=: E^{i+j} .
$$

We have

$$
\begin{aligned}
& \mu_{*} \mathcal{O}_{V}(M) \\
= & \mu_{*} \mathcal{O}_{V}\left(\left\ulcorner\mu^{*}(D)+K_{V}-\mu^{*}\left(K_{X}+\Delta\right)\right\urcorner\right) \\
= & \mu_{*} \mathcal{O}_{V}\left(\left\llcorner\mu^{*}(D)\right\lrcorner+(\text { effective exceptional } \mathbb{Z} \text {-divisor })\right) \\
\simeq & \mathcal{O}_{X}(D)
\end{aligned}
$$

where the second equality holds because $(X, \Delta)$ is klt and $D$ is a $\mathbb{Z}$ divisor. Since $E_{2}^{i, j}=0$ for $j>0$ by the relative Kawamata-Viehweg vanishing theorem for a proper birational morphism between surfaces, we obtain

$$
H^{i}\left(X, \mathcal{O}_{X}(D)\right)=E_{2}^{i, 0} \simeq E^{i}=H^{i}\left(V, \mathcal{O}_{V}(M)\right)=0
$$

for $i>0$. Thus, the claim follows.

Proof of Theorem 1.2. After possibly perturbing $B$ and $\Delta$, we may assume that $-\left(K_{X}+B\right)$ and $D-\left(K_{X}+\Delta\right)$ are ample. Given $I:=\emptyset$, let $p_{0}:=p(I)$ be the positive integer whose existence is guaranteed by Theorem 1.1. We divide the proof into five steps:

Step 1. We first prove the Theorem assuming that $\Delta=B=0$. 
Theorem 1.1 implies that $X$ is globally $F$-regular or $X$ admits a log resolution $\mu: V \rightarrow X$ such that $(V, \operatorname{Exc}(f))$ lifts to $W_{2}(k)$. In the first case, by Serre vanishing, if $e$ is a sufficiently large positive integer and $i>0$, we have

$$
\begin{aligned}
H^{i}\left(X, \mathcal{O}_{X}(D)\right) & \hookrightarrow H^{i}\left(X, F_{*}^{e} \mathcal{O}_{X}\left(p^{e} D-\left(p^{e}-1\right) K_{X}\right)\right) \\
& \simeq H^{i}\left(X, \mathcal{O}_{X}\left(D+\left(p^{e}-1\right)\left(D-K_{X}\right)\right)\right)=0 .
\end{aligned}
$$

On the other hand, if $X$ admits a $\log$ resolution $\mu: V \rightarrow X$ such that $(V, \operatorname{Exc}(f))$ lifts to $W_{2}(k)$, then Lemma 6.1 implies the claim.

Step 2. We now prove the Theorem under the assumption that $D$ is nef.

Let $f: X \rightarrow Y$ be a proper birational contraction obtained by running a $\left(-K_{X}\right)$-MMP. Since $(X, B)$ is log del Pezzo, it follows that $Y$ is klt and $-K_{Y}$ is big and semi-ample. Moreover, there exists an $f$ exceptional $\mathbb{Q}$-divisor $\Delta_{1} \geq 0$ such that

$$
K_{X}+\Delta_{1}=f^{*} K_{Y}
$$

Let $g: Y \rightarrow Z$ be the morphism induced by $-K_{Y}$ and let

$$
h: X \stackrel{f}{\rightarrow} Y \stackrel{g}{\rightarrow} Z
$$

be the composite morphism. Then $-K_{Z}$ is ample and

$$
K_{X}+\Delta_{1}=h^{*} K_{Z}
$$

By Theorem 1.1, $Z$ is globally $F$-regular or it admits a log resolution $\mu: V \rightarrow Z$ such that $(V, \operatorname{Exc}(\mu))$ lifts to characteristic zero over a smooth base.

If $Z$ is globally $F$-regular, then [HX15, Proposition 2.11] implies that $\left(X, \Delta_{1}\right)$ is also globally $F$-regular. Thus, we may find a $\mathbb{Q}$-divisor $\Delta_{2} \geq \Delta_{1}$ such that $\left(X, \Delta_{2}\right)$ is globally $F$-regular, $-\left(K_{X}+\Delta_{2}\right)$ is ample, and $\left(p^{e_{1}}-1\right)\left(K_{X}+\Delta_{2}\right)$ is Cartier for some $e_{1} \in \mathbb{Z}_{>0}$. Since $D$ is nef, by Fujita vanishing, if $e$ is a sufficiently divisible positive integer, for any $i>0$ we have

$$
H^{i}\left(X, \mathcal{O}_{X}(D)\right) \hookrightarrow H^{i}\left(X, F_{*}^{e} \mathcal{O}_{X}\left(p^{e} D-\left(p^{e}-1\right)\left(K_{X}+\Delta_{2}\right)\right)\right)=0,
$$

and the Theorem follows.

Thus, we may assume the existence of the $\log$ resolution $\mu$, as above. Since $\Delta_{1} \geq 0$, the birational morphism $h: X \rightarrow Z$ only extracts prime divisors $E$ with $a(E, Z, 0) \in(0,1]$. By Remark 4.4, we may assume that $\mu: V \rightarrow Z$ factors through $X$, after possibly replacing $V$ by some blow-up of $V$ :

$$
\mu: V \underset{32}{\stackrel{\mu_{X}}{\rightarrow}} X \stackrel{h}{\rightarrow} Z
$$


There exists an $h$-exceptional $\mathbb{Q}$-divisor $\Delta_{2} \geq \Delta_{1}$ such that $\left(X, \Delta_{2}\right)$ is klt and $-\left(K_{X}+\Delta_{2}\right)$ is ample. Since $\mu_{X}^{-1}\left(\Delta_{2}\right) \subseteq \operatorname{Exc}(\mu)$, Lemma 6.1 implies the Theorem.

Step 3. We now show that we may assume that there exists a $D$ negative Mori fibre space $g: X \rightarrow Z$ onto a smooth projective curve $Z$.

Let $f: X \rightarrow Y$ be the birational contraction of a curve $E$ such that $D \cdot E \leq 0$. We want to show

$$
H^{i}\left(X, \mathcal{O}_{X}(D)\right)=H^{i}\left(Y, \mathcal{O}_{Y}\left(f_{*} D\right)\right) .
$$

Let $D_{Y}=f_{*} D$. Then $D=f^{*} D_{Y}+c E$ for some $c \in \mathbb{Q}_{\geq 0}$, which implies

$$
f_{*} \mathcal{O}_{X}(D)=\mathcal{O}_{Y}\left(D_{Y}\right) \text {. }
$$

Since $D-\left(K_{X}+\Delta\right)$ is ample, the relative Kawamata-Viehweg vanishing in the birational case implies

$$
H^{i}\left(X, \mathcal{O}_{X}(D)\right) \simeq H^{i}\left(Y, f_{*} \mathcal{O}_{X}(D)\right)=H^{i}\left(Y, \mathcal{O}_{Y}\left(D_{Y}\right)\right) .
$$

Since $(X, B)$ is log del Pezzo, we may run a $D$-MMP and may assume that one of the following holds:

- $D$ is nef,

- $\rho(X)=2$, and there exists a $D$-negative Mori fibre space $X \rightarrow$ $Z$ onto a smooth projective curve $Z$, or

- $\rho(X)=1$, and $-D$ is ample.

If $D$ is nef, then we may apply Step 2. In the last case, we may assume that $\Delta=B=0$, and we may apply Step 1 . Thus, we may assume that there exists a $D$-negative Mori fibre space $g: X \rightarrow Z$ onto a smooth projective curve $Z$.

Step 4. We now show that we may assume that the following hold:

(a) There exists a curve $E$ on $X$ such that $E^{2}<0$,

(b) $B=b E$ for some $b \in[0,1)$, and

(c) $\Delta=0$.

If every curve $C$ on $X$ is nef, then we may assume that $\Delta=B=0$, and apply Step 1 . Thus, we may assume that there exists a curve $E$ on $X$ such that $E^{2}<0$ and (a) holds.

By Step 3, we may assume that there exists a $D$-negative Mori fibre space $g: X \rightarrow Z$ onto a smooth projective curve $Z$. In particular, $\rho(X)=2$, and any curve $C$ on $X$, which is different from $E$, is nef. Thus, we may assume that (b) holds. Similarly, we may assume that 
$\Delta=\delta E$ for some $\delta \in[0,1)$. Let $F$ be a general fibre of $g$. Then $D \cdot F<0$. Consider the short exact sequence

$$
0 \rightarrow \mathcal{O}_{X}(D) \rightarrow \mathcal{O}_{X}(D+F) \rightarrow \mathcal{O}_{F}\left(\left.D\right|_{F}\right) \rightarrow 0 .
$$

Since $H^{0}\left(F, \mathcal{O}_{F}\left(\left.D\right|_{F}\right)\right)=H^{1}\left(F, \mathcal{O}_{F}\left(\left.D\right|_{F}\right)\right)=0$, it is enough to show that $H^{i}\left(X, \mathcal{O}_{X}(D+F)\right)=0$ for any $i>0$. Repeating the same method finitely many times, it is enough to show that $H^{i}\left(X, \mathcal{O}_{X}(D+n F)\right)=0$ for some positive integer $n$ and for any $i>0$. Since $D-\left(K_{X}+\delta E\right)$ is ample, the divisor

$$
D+n F-K_{X}=\left(D-\left(K_{X}+\delta E\right)\right)+(n F+\delta E)
$$

is also ample for $n \gg 0$. Therefore, after possibly replacing $D$ by $D+n F$, we may assume that $\Delta=0$ and (c) holds.

Step 5. We now prove the Theorem in the general case.

We may assume that (a), (b) and (c) of Step 4 hold. If $K_{X} \cdot E<0$, then $-K_{X}$ is ample by Kleiman's criterion and we may assume that $B=0$. Thus, the Theorem follows by Step 1 .

Therefore, we may assume that $K_{X} \cdot E \geq 0$. Let $f: X \rightarrow Y$ be the birational morphism which contracts $E$. We may write

$$
K_{X}+b^{\prime} E=f^{*} K_{Y}
$$

for some $b^{\prime} \in[0, b)$. Thus, by Theorem 1.1 and Remark 4.4, $Y$ is globally $F$-regular or it admits a $\log$ resolution $V \rightarrow Y$ which factors through $X$ such that $(V, \operatorname{Exc}(\mu))$ lifts to $W_{2}(k)$ :

$$
\mu: V \stackrel{\mu_{X}}{\longrightarrow} X \rightarrow Y .
$$

In the latter case, since $\operatorname{Exc}\left(\mu_{X}\right) \subseteq \operatorname{Exc}(\mu)$, we may apply Lemma 6.1. Thus, we may assume that $Y$ is globally $F$-regular. By [HX15, Prop $2.11],\left(X, b^{\prime} E\right)$ is globally $F$-regular, and so is $X$. Step 4(c) implies that $D-K_{X}$ is ample, Therefore, by Serre vanishing, if $e$ is a sufficiently large positive integer, we have

$H^{i}(X, D) \hookrightarrow H^{i}\left(X, p^{e} D-\left(p^{e}-1\right) K_{X}\right)=H^{i}\left(X, K_{X}+p^{e}\left(D-K_{X}\right)\right)=0$.

Thus, the Theorem follows.

\section{EXAMPle IN CHARACTERISTIC TWO}

The goal of this section is to show Theorem 1.3. We begin with the following two preliminary results: 
Lemma 7.1. Let $T \rightarrow$ Spec $\mathbb{Z}$ be a smooth morphism from an integral scheme $T$ and let $\mathcal{W} \rightarrow T$ be a flat projective morphism. Fix a morphism $\alpha$ : Spec $\overline{\mathbb{F}}_{p} \rightarrow T$ and an embedding $K(T) \hookrightarrow \mathbb{C}$. Let $W_{\overline{\mathbb{F}}_{p}}:=\mathcal{W} \times_{T} \overline{\mathbb{F}}_{p}$, and $W_{\mathbb{C}}:=\mathcal{W} \times_{T} \mathbb{C}$. If $W_{\overline{\mathbb{F}}_{p}}$ is a smooth projective rational surface, then the following hold:

(1) $W_{\mathbb{C}}$ is a smooth projective rational surface, and

(2) $\rho\left(W_{\mathbb{C}}\right)=\rho\left(W_{\overline{\mathbb{F}}_{p}}\right)$.

Proof. Since being smooth and geometrically integral are open properties, it follows that $W_{\mathbb{C}}$ is a smooth projective surface.

By upper semi-continuity, it follows that $H^{i}\left(W_{\mathbb{C}}, \mathcal{O}_{W_{\mathbb{C}}}\right)=0$ for any $i>0$ and $H^{2}\left(W_{\mathbb{C}}, \mathcal{O}_{W_{\mathbb{C}}}\left(-K_{W_{\mathbb{C}}}\right)\right)=0$. Thus, by the rationality criterion, it follows that $W_{\mathbb{C}}$ is a rational surface and (1) holds.

Note that, for any sufficiently large positive integer $e$, the morphism $\alpha$ factors as follows

$$
\alpha: \text { Spec } \overline{\mathbb{F}}_{p} \rightarrow \text { Spec } \mathbb{F}_{p^{e}} \rightarrow T \text {. }
$$

Let $W_{\mathbb{F}_{p^{e}}}:=\mathcal{W} \times_{T} \mathbb{F}_{p^{e}}$. After possibly replacing $e$ by a larger number, we may assume that $W_{\mathbb{F}_{p} e}$ is obtained as a sequence of blow-ups of $\mathbb{P}_{\mathbb{F}_{p}}^{2}$ or a $\mathbb{P}^{1}$-bundle over $\mathbb{P}_{\mathbb{F}_{p} e}^{1}$, whose centres are $\mathbb{F}_{p^{e}}$-rational points. By counting the rational points, it is easy to check that the zeta function of $W_{\mathbb{F}_{p} e}$ can be written as

$$
Z_{W_{\mathbb{F}_{p}}}(t)=\frac{1}{(1-t)\left(1-p^{e} t\right)^{\rho\left(W_{\mathbb{F}_{p}}\right)}\left(1-p^{2 e} t\right)} .
$$

On the other hand, we obtain

$$
\rho\left(W_{\overline{\mathbb{F}}_{p}}\right)=\operatorname{deg}\left(1-p^{e} t\right)^{\rho\left(W_{\overline{\mathbb{F}}_{p}}\right)}=\operatorname{dim}_{\mathbb{C}} H^{2}\left(W_{\mathbb{C}}, \mathbb{C}\right)=\rho\left(W_{\mathbb{C}}\right),
$$

where the second equality follows from a consequence of the Weil conjecture [FK88, Chapter IV, Remark (b) after Theorem 1.2]. Thus, (2) holds.

Lemma 7.2. Let $k$ be an algebraically closed field of characteristic two. Then there exist six distinct points $q_{0}, \ldots, q_{5} \in \mathbb{P}_{k}^{2}$ which satisfy the following properties:

(1) if $g: Z \rightarrow \mathbb{P}_{k}^{2}$ is the blow-up at $q_{0}, \ldots, q_{5}$, then $Z$ is a smooth del Pezzo surface,

(2) $Z$ is not globally F-split, and

(3) if $C$ is the smooth conic passing through $q_{1}, \ldots, q_{5}$, then, for every point $q \in C$, the line $L$ passing through $q_{0}$ and $q$ is tangent to $C$. 
Proof. The smooth cubic surface $Z$ of Fermat type is not globally $F$ split [Har98, Example 5.5]. We can find six points $q_{0}, \ldots, q_{5} \in \mathbb{P}_{k}^{2}$ such that the blow-up along these points is isomorphic to $Z$. Let $g: Z \rightarrow \mathbb{P}_{k}^{2}$ be the induced morphism. Then (1) and (2) hold.

By contradiction, we assume that there exists a line $L$ on $\mathbb{P}_{k}^{2}$, passing through $q_{0}$, such that $C+L$ is simple normal crossing. Let $C_{Z}$ and $L_{Z}$ be the proper transforms on $Z$ of $C$ and $L$, respectively. By (1), there exists a point $q \in C \cap L$ with $q \neq q_{i}$ for any $i=1, \ldots, 5$, and, in particular, $C_{Z} \cap L_{Z} \neq \emptyset$. There exists a reduced $g$-exceptional divisor $E_{Z}$ on $Z$ such that

$$
K_{Z}+C_{Z}+L_{Z}+E_{Z}=g^{*}\left(K_{\mathbb{P}_{k}^{2}}+C+L\right) \sim 0,
$$

and $\left(Z, C_{Z}+L_{Z}+E_{Z}\right)$ is log canonical but not plt. By Lemma 2.9. $\left(Z, C_{Z}+L_{Z}+E_{Z}\right)$ is globally $F$-split. Thus, $Z$ is globally $F$-split, contradicting (2). Thus, (3) holds.

We now prove the main result of this section.

Proof of Theorem 1.3. Let $q_{0}, \ldots, q_{5} \in \mathbb{P}_{\mathbb{F}_{2}}^{2}$ be as in Lemma 7.2. For any $i=1, \ldots, 5$, let $L_{i}$ be the line in $\mathbb{P}_{\mathbb{F}_{2}}^{2}$ passing through $q_{0}$ and $q_{i}$. Note that each $L_{i}$ is tangent to $C$ by (3) of Lemma 7.2. Let

$$
\pi: Y \rightarrow \mathbb{P}_{\mathbb{F}_{2}}^{2}
$$

be the birational morphism constructed as follows. First, we consider the blow-up $Z \rightarrow \mathbb{P}_{\mathbb{F}_{2}}^{2}$ at the points $q_{0}, \ldots, q_{5}$. Then, we consider the blow-up $Y \rightarrow Z$ at the points $q_{1}^{\prime}, \ldots, q_{5}^{\prime}$, where $q_{i}^{\prime}$ is the intersection point of the proper transforms on $Z$ of $C$ and $L_{i}$. In particular, $\rho(Y)=$ 12. By the same argument as in [KM99, Section 9, An interesting example in non-zero characteristic], we can find curves $E_{2}, E_{3}, \ldots, E_{12}$ on $Y$ with

$$
E_{2}^{2}=-6, \quad E_{m}^{2}=-2, \quad E_{i} \cdot E_{j}=0
$$

for any $m=3, \ldots, 12$ and distinct $i, j=2, \ldots, 12$. Let $f: Y \rightarrow X$ be the birational morphism which contracts all these curves. Then $X$ is a projective klt surface with $\rho(X)=1$. By [ibid], $-K_{X}$ is ample. Thus, (1) holds.

By (2) of Lemma 7.2, $Z$ is not globally $F$-split. By CTW16, Lemma 2.2 ], it follows that $Y$ is not globally $F$-split and by [HX15, Proposition 2.11], it follows that $X$ is not globally $F$-split. Thus, (2) holds.

We now show (3). By contradiction, we assume that there exists a $\log$ resolution $h: W \rightarrow X$ such that $\left(W, \operatorname{Exc}(h)=\sum_{i=2}^{n} C_{i}\right)$ lifts to characteristic zero over a smooth base, i.e. there exists a smooth morphism $T \rightarrow$ Spec $\mathbb{Z}$, a closed immersion of schemes $\mathcal{C}_{i} \subseteq \mathcal{W}$ projective 
and flat over $T$ for any $i=2, \ldots, n$, and a morphism $\alpha$ : Spec $\overline{\mathbb{F}}_{2} \rightarrow T$ such that the base changes of $\mathcal{C}_{i} \subseteq \mathcal{W}$ by $\alpha$ is $C_{i} \subseteq W$ and such that $\mathcal{W}$ and all the strata of $\left\{\mathcal{C}_{i}\right\}$ are smooth over $T$. We may assume that $T$ is an integral scheme. Note that $K(T)$ is of characteristic zero. Fix an embedding $K(T) \hookrightarrow \mathbb{C}$, and let $W_{\mathbb{C}}:=\mathcal{W} \times_{T} \mathbb{C}$. By Lemma 7.1, we have

$$
n=\rho(W)=\rho\left(W_{\mathbb{C}}\right) .
$$

The birational morphism $f: Y \rightarrow X$ constructed above is the minimal resolution of $X$. Thus, $h$ factors as

$$
h: W \stackrel{g}{\rightarrow} Y \stackrel{f}{\rightarrow} X
$$

and may assume that $g_{*} C_{i}=E_{i}$ for any $i=2, \ldots, 12$. We can find a sequence of blow-ups

$$
g: W=: W_{n} \stackrel{g_{n}}{\longrightarrow} W_{n-1} \stackrel{g_{n-1}}{\longrightarrow} \ldots \stackrel{g_{13}}{\longrightarrow} W_{12}:=Y
$$

with $\rho\left(W_{r}\right)=r$ for any $r=12, \ldots, n$. We may assume that the proper transform of $\operatorname{Exc}\left(g_{r}\right)$ on $W$ is $C_{r}$.

By invariance of the intersection numbers, the two intersection matrices $\left(C_{i} \cdot C_{j}\right)$ and $\left(C_{i, \mathbb{C}} \cdot C_{j, \mathbb{C}}\right)$ coincide. Thus, we can construct the corresponding sequence over $\mathbb{C}$, i.e. a sequence of blow-ups:

$$
g_{\mathbb{C}}: W_{\mathbb{C}}=: W_{n, \mathbb{C}} \stackrel{g_{n, \mathbb{C}}}{\longrightarrow} W_{n-1, \mathbb{C}} \stackrel{g_{n-1, \mathbb{C}}}{\longrightarrow} \ldots \stackrel{g_{13, \mathbb{C}}}{\longrightarrow} W_{12, \mathbb{C}}:=Y_{\mathbb{C}}
$$

such that $\rho\left(W_{r, \mathbb{C}}\right)=r$ for every $r=12, \ldots, n$ and such that the proper transform of $\operatorname{Exc}\left(g_{r, \mathbb{C}}\right)$ on $W_{\mathbb{C}}$ is $C_{r, \mathbb{C}}$. Indeed, it is easy to check that the push-forward of $C_{i, \mathbb{C}}$ on $W_{i, \mathbb{C}}$ is a $(-1)$-curve and that the curves $E_{2, \mathbb{C}}:=\left(g_{\mathbb{C}}\right)_{*} C_{2, \mathbb{C}}, \ldots, E_{12, \mathbb{C}}:=\left(g_{\mathbb{C}}\right)_{*} C_{12, \mathbb{C}}$ satisfy

$$
E_{2, \mathbb{C}}^{2}=-6, \quad E_{m, \mathbb{C}}^{2}=-2, \quad E_{i, \mathbb{C}} \cdot E_{j, \mathbb{C}}=0
$$

for any $m=3, \ldots, 12$ and distinct $i, j=2, \ldots, 12$. Note also that $E_{\ell, \mathbb{C}}$ is isomorphic to $\mathbb{P}_{\mathbb{C}}^{1}$ for any $\ell=2, \ldots, 12$. Let

$$
f_{\mathbb{C}}: Y_{\mathbb{C}} \rightarrow X_{\mathbb{C}}
$$

be the birational morphism which contracts $E_{2, \mathbb{C}}, \ldots, E_{12, \mathbb{C}}$. Then $X_{\mathbb{C}}$ is a projective klt surface with exactly 11 singular points. Moreover, since $\rho\left(W_{\mathbb{C}}\right)=n$, it follows that $\rho\left(X_{\mathbb{C}}\right)=1$.

Further, for any invertible sheaf $\mathcal{A}$ on $\mathcal{W}$ which is ample over $T$, we have

$$
0>\left.g^{*} f^{*} K_{X} \cdot \mathcal{A}\right|_{W}=\left.g_{\mathbb{C}}^{*} f_{\mathbb{C}}^{*} K_{X_{\mathbb{C}}} \cdot \mathcal{A}\right|_{W_{\mathbb{C}}}
$$

Thus, $-K_{X_{\mathbb{C}}}$ is ample. On the other hand, [KM99, Theorem 9.2] implies that $X_{\mathbb{C}}$ admits at most 6 singularities, a contradiction. Thus, (3) holds. 


\section{REFERENCES}

[Ale93] V. Alexeev, Two two-dimensional terminations, Duke Math. J. 69 (1993), no. 3, 527-545.

[Ale94] Boundedness and $K^{2}$ for log surfaces, International J. Math. 5 (1994), 779-810.

[Băd01] L. Bădescu, Algebraic surfaces, Universitext, Springer-Verlag, New York, 2001.

[Bir16] C. Birkar, Existence of flips and minimal models for 3-folds in char p, Ann. Sci. Ecole Norm. Sup. 49 (2016), no. 1, 169-212.

[BW14] C. Birkar and J. Waldron, Existence of Mori fibre spaces for 3-folds in char p, arXiv:1410.4511 (2014).

[CGS16] P. Cascini, Y. Gongyo, and K. Schwede, Uniform bounds for strongly Fregular surfaces, Trans. Amer. Math. Soc. 368 (2016), no. 8, 5547-5563.

[CTW16] P. Cascini, H. Tanaka, and J. Witaszek, Klt del Pezzo surfaces which are not globally F-split, arXiv:1601.03578 (2016).

[CTX15] P. Cascini, H. Tanaka, and $\mathrm{C}$. Xu, On base point freeness in positive characteristic, Ann. Sci. Ecole Norm. Sup. 48 (2015), no. 5, 1239-1272.

[Das15] O. Das, On strongly F-regular inversion of adjunction, J. Algebra 434 (2015), 207-226.

[EV92] H. Esnault and E. Viehweg, Lectures on vanishing theorems, DMV Seminar, vol. 20, Birkhäuser Verlag, Basel, 1992.

$\left[\mathrm{FGI}^{+} 05\right]$ B. Fantechi, L. Göttsche, L. Illusie, S. L. Kleiman, N. Nitsure, and A. Vistoli, Fundamental algebraic geometry, American Mathematical Society, Providence, RI, 2005.

[FK88] E. Freitag and R. Kiehl, Étale cohomology and the Weil conjecture, Ergebnisse der Mathematik und ihrer Grenzgebiete (3) [Results in Mathematics and Related Areas (3)], vol. 13, Springer-Verlag, Berlin, 1988.

[Fu15] L. Fu, Etale cohomology theory, revised ed., Nankai Tracts in Mathematics, vol. 14, World Scientific Publishing Co. Pte. Ltd., Hackensack, NJ, 2015 .

[Fuj12] O. Fujino, Minimal model theory for log surfaces, Publ. Res. Inst. Math. Sci. 48 (2012), no. 2, 339-371.

[Har98] N. Hara, A characterization of rational singularities in terms of injectivity of Frobenius maps, Amer. J. Math. 120 (1998), no. 5, 981-996.

[HX15] C. Hacon and C. Xu, On the three dimensional minimal model program in positive characteristic, J. Amer. Math. Soc. 28 (2015), no. 3, 711-744.

$\left[\mathrm{K}^{+} 92\right] \quad J$. Kollár et al., Flips and abundance for algebraic threefolds, Société Mathématique de France, Paris, 1992.

[KM98] J. Kollár and S. Mori, Birational Geometry of Algebraic Varieties, Cambridge Tracts in Mathematics, vol. 134, Cambridge University Press, 1998.

[KM99] S. Keel and J. McKernan, Rational curves on quasi-projective surfaces, Mem. Amer. Math. Soc. 140 (1999), no. 669, viii+153.

[Kol13] J. Kollár, Singularities of the minimal model program, Cambridge Tracts in Mathematics, vol. 200, Cambridge University Press, Cambridge, 2013.

[Lan16] A. Langer, The Bogomolov-Miyaoka-Yau inequality for logarithmic surfaces in positive characteristic, Duke Math. J. 165 (2016), no. 14, 27372769 . 
[Liu02] Q. Liu, Algebraic geometry and arithmetic curves, Oxford Graduate Texts in Mathematics, vol. 6, Oxford University Press, Oxford, 2002.

[MP04] J. M'Kernan and Y. Prokhorov, Threefold Thresholds, Manuscripta Math. 114 (2004), no. 3, 281-304.

[Pro01] Y. Prokhorov, Lectures on complements on log surfaces, MSJ Memoirs, vol. 10, Mathematical Society of Japan, Tokyo, 2001.

[Sch09] K. Schwede, F-adjunction, Algebra Number Theory 3 (2009), no. 8, 907950 .

[Sch14] , A canonical linear system associated to adjoint divisors in characteristic $p>0$, J. Reine Angew. Math. 696 (2014), 69-87.

[SS10] K. Schwede and K. E. Smith, Globally F-regular and log Fano varieties, Adv. Math. 224 (2010), no. 3, 863-894.

[Tan14] H. Tanaka, Minimal models and abundance for positive characteristic log surfaces, Nagoya Math. J. 216 (2014), 1-70.

[Tan15] _ , The $X$-method for klt surfaces in positive characteristic, J. Algebraic Geom. 24 (2015), no. 4, 605-628.

[Wat91] K. Watanabe, F-regular and F-pure normal graded rings, J. Pure Appl. Algebra 71 (1991), no. 2-3, 341-350.

[Wit15] J. Witaszek, Effective bounds on singular surfaces in positive characteristic, Michigan Math. J. (to appear) (2015).

Department of Mathematics, Imperial College, London, 180 Queen's Gate, London SW7 2AZ, UK

E-mail address: p.cascini@imperial.ac.uk

E-mail address: h.tanaka@imperial.ac.uk

E-mail address: j.witaszek14@imperial.ac.uk 